\title{
Magnetic turbulence in cool cores of galaxy clusters ${ }^{\star}$
}

\author{
T. A. Enßlin ${ }^{1}$ and C. $\operatorname{Vogt}^{1,2}$ \\ 1 Max-Planck-Institut für Astrophysik, Karl-Schwarzschild-Str.1, Postfach 1317, 85741 Garching, Germany \\ 2 ASTRON, PO Box 2, 7990 AA Dwingeloo, The Netherlands
}

Received 25 May 2005 / Accepted 9 March 2006

ABSTRACT

\begin{abstract}
We argue that the recently reported Kolmogorov-like magnetic turbulence spectrum in the cool core of the Hydra A galaxy cluster can be understood by kinetic energy injection by active galaxies that drives a turbulent non-helical magnetic dynamo into its saturated state. Although dramatic differences exist between small-scale dynamo scenarios, their saturated state is expected to be similar, as we show for three scenarios: the flux rope dynamo, the fluctuation dynamo, and the explosive dynamo. Based on those scenarios, we develop an analytical model of the hydrodynamic and magnetic turbulence in cool cores. The model implies magnetic field strengths that fit well with Faraday rotation measurements and minimum energy estimates for the sample of cool core clusters having such data available. Predictions for magnetic fields in clusters for which the appropriate observational information is still missing, and for yet unobserved quantities like the hydrodynamical turbulence velocity and characteristic length-scale are provided. The underlying dynamo models suggest magnetic intermittency and possibly a large-scale hydrodynamic viscosity. We conclude that the success of the model to explain the field strength in cool core clusters indicates that in general cluster magnetic fields directly reflect hydrodynamical turbulence, also in clusters without cool cores.
\end{abstract}

Key words. galaxies: cluster: general - cooling flows - magnetic fields - turbulence - X-rays: galaxies: clusters intergalactic medium

\section{Introduction}

\subsection{Magnetic fields in cool cores}

Galaxy clusters contain magnetised plasma on cluster scales, as radio-synchrotron emission of relativistic electrons reveals in form of the so-called cluster radio halos. The origin, the strength and geometry of these magnetic fields is still a mystery.

With the emerging of the first theories of magnetic dynamos (Batchelor 1950; Kazantsev 1967; Zeldovich et al. 1990), it was proposed that the cluster magnetic fields are the product of turbulence acting on a seed magnetic field. The seed field could be a remnant of non-equilibrium processes in the early Universe, or due to some weak magnetisation caused by galactic outflows in the form of winds and radio plasma (for a review see Widrow 2002).

Initially, wakes of the weakly sonic galaxy motions were thought to be the main driver of the intra-cluster turbulence ${ }^{1}$. However, it was realised using numerical simulations of largescale structure formation that the violent mergers of galaxy clusters are a much more powerful source of turbulence (e.g. Tribble 1993). In numerical simulations of magnetic field amplification, the merger-driven turbulence of galaxy clusters seems to be able to reproduce the typical field values of clusters ${ }^{2}$.

It is therefore surprising that the strongest magnetic fields seem to be located in the centres of clusters which are dynamically the most relaxed, since the last major merger was long ago. These cooling flow (now cool core) clusters had time to

\footnotetext{
* Appendix is only available in electronic form at http://www . edpsciences.org

1 e.g. Jaffe (1980); Roland (1981); Ruzmaikin et al. (1989); Goldman \& Rephaeli (1991); De Young (1992).

2 Dolag et al. (1999); Roettiger et al. (1999b); Dolag et al. (2002).
}

develop a cool, dense central region due to the cooling instability of optically thin X-ray emission of cooling gas. The magnetic fields reported for these cooling flow regions inferred by Faraday rotation studies were extraordinarily strong (up to $50 \mu \mathrm{G}$ ) compared to the few $\mu \mathrm{G}$ fields reported for non-cooling flow clusters (for recent reviews see Carilli \& Taylor 2002; Govoni \& Feretti 2004). It was speculated that the strong fields could be a result of compression in the cooling flow (Soker \& Sarazin 1990).

However, Chandra and XMM observations revealed that the standard cooling flow picture, in which unheated gas cools down to neutral gas temperatures, must be incorrect, since the expected amount of line emission of cold $(<0.3 \mathrm{keV})$ gas or the expected number of stars formed from the condensing gas was not detected $^{3}$. Therefore a heat source has to be present which balances the cooling of the coldest gas. Since the theoretical scenario associated with the term cooling flow has been ruled out recently, the only observationally motivated term cool core is used in the following.

The energy losses of the cool core have to be balanced by a similar energy injection. There have been two main proposals ${ }^{4}$ for the heat source in cool cores:

(i) Thermal conductivity, which is close to Spitzer's estimate, and therefore not suppressed by magnetic fields. This would

\footnotetext{
${ }^{3}$ Heckman et al. (1989); Fabian et al. (1991); Hansen et al. (1995); Allen (1995); Jaffe \& Bremer (1997); Smith et al. (1997); O’Dea et al. (1998); Crawford et al. (1999); Donahue et al. (2000); Edge (2001); Oegerle et al. (2001); Salomé \& Combes (2003); Edge \& Frayer (2003).

${ }_{4}$ There are also other mechanisms discussed in the literature, e.g. the influence of a cosmic ray population (Chandran 2004; Cen 2005).
} 
allow the inward transport of heat from the hotter environmental intra-cluster medium (ICM) ${ }^{5}$.

(ii) Dissipation of mechanical energy released by the expansion and buoyant motion of radio bubbles inflated by the radio galaxies which are typically found in the centre of a cool core of a galaxy cluster ${ }^{6}$.

The thermal conduction scenario (i) as the only heat injection mechanism into cool cores faces severe problems in terms of fine-tuning the required energy injection, and explaining the existence of cold gas clouds which need sufficient insulation from the keV gas (Soker 2003; Nipoti \& Binney 2004). Too strong conduction would erase the cool core, but too weak conduction cannot prevent the cooling catastrophe. Therefore a conductively heated cool core should be unstable.

The scenario (ii) in which the central radio galaxy balances the radiative energy losses of the cool core provides fine tuning in the form of a self-adapting feedback mechanism: if the radio galaxy activity is triggered by cold gas condensing out of the cool core onto the central galaxy, the galaxy activity increases until it disrupts further accretion (Churazov et al. 2001).

In this work we assume scenario (ii), not only since it is at least in our view - theoretically more compelling, but also because it predicts a certain level of hydrodynamical turbulence, which can be compared to the level required to explain the cool core magnetic fields by magnetic dynamo theory.

There have been some reports on observed signatures of turbulent flows in cool cores of galaxy clusters, and the scenario (ii) investigated here seems to become widely accepted, at least as a working hypothesis (e.g. Loewenstein \& Fabian 1990; Churazov et al. 2001; Böhringer et al. 2002; Churazov et al. 2002, 2004).

\subsection{Observations of cluster magnetic fields}

Magnetic fields in normal clusters and cool core clusters have revealed their existence by the diffuse radio halo emission in many clusters and radio mini-halos in cool core regions. Furthermore, the Faraday rotation of linearly polarised radio emission traversing the intra-cluster medium independently proves the existence of intra-cluster magnetic fields. If the Faraday active medium is external to the source of the polarised emission, one expects the change in polarisation angle to be proportional to the squared wavelength. The proportionality factor is called the rotation measure $(R M)$. This quantity can be evaluated in terms of the line of sight integral over the product of the electron density and the magnetic field component along the line of sight.

Magnetic fields in non-cool core clusters of galaxies detected through Faraday rotation measurements are of the order of a few $\mu \mathrm{G}$. Kim et al. (1991) measured field strengths of about $2 \mu \mathrm{G}$ on scales of $10 \mathrm{kpc}$ in a statistical sample of point sources observed through the Coma cluster. Clarke et al. (2001), Clarke (2004), and Johnston-Hollitt \& Ekers (2004) derive similar field strengths of several $\mu \mathrm{G}$ in various samples of point sources observed within and through various low redshift clusters in the

\footnotetext{
${ }^{5}$ Malyshkin (2001); Narayan \& Medvedev (2001); Voigt et al. (2002); Ruszkowski \& Begelman (2002); Cho et al. (2003); Soker et al. (2004); Voigt \& Fabian (2004); Soker (2004); Jubelgas et al. (2004); Chandran \& Maron (2004); Dolag et al. (2004).

${ }^{6}$ Churazov et al. (2001); Brüggen \& Kaiser (2001); Quilis et al. (2001); Brüggen et al. (2002); Chandran (2004); Hoeft \& Brüggen (2004); Dennis \& Chandran (2005). There have been also observations of hot gas bubbles (Mazzotta et al. 2004), which may also contribute to the cool core heating in a very similar way as the radio bubbles (Soker \& Pizzolato 2005).
}

northern and southern hemisphere. The analysis of $R M$ maps of extended radio sources have lead to the same conclusion (e.g. Feretti et al. 1995, 1999; Taylor et al. 2001; Govoni et al. 2001; Eilek \& Owen 2002; Vogt \& Enßlin 2003).

However, the analysis of Faraday rotation measurements of extended radio sources in the centre of cool core clusters reveal higher magnetic field strengths. Such an analysis has been done for the Centaurus cluster by Taylor et al. (2002), for A1958 (better known as 3C 295) by Perley \& Taylor (1991), for A1795 by Ge \& Owen (1993), for Cygnus A by Dreher et al. (1987) and for Hydra A by Taylor \& Perley (1993). From these RM measurements, magnetic field strengths of 10 to $40 \mu \mathrm{G}$ have been reported for the cores of these cool core clusters on scales of 3-5 kpc.

These rather large field values for cool core clusters have been revised in the case of Hydra A by a recent analysis of the observational data. A high quality Faraday rotation map of the north lobe of Hydra A produced by the novel PACERMAN algorithm (Dolag et al. 2005; Vogt et al. 2005) based on the data of Taylor \& Perley (1993) was analysed by Vogt \& Enßlin (2005). They used a maximum likelihood estimator for the derivation of the magnetic power spectra, based on the theory of turbulent Faraday screens (Enßlin \& Vogt 2003; Vogt \& Enßlin 2003), and also using the most up-to date gas density profile of the cool core, which turned out to make a crucial difference. Thereby, a magnetic field strength of $7 \pm 2 \mu \mathrm{G}$ was found in the centre of the cool core region of the Hydra A cluster, which is still a significantly larger field than reported for non-cool core clusters. Vogt \& Enßlin (2005) measured the detailed magnetic power spectrum from the Hydra A dataset, which revealed a Kolmogorovtype spectrum on small scales indicating turbulence (see Fig. 1).

It has been debated whether the magnetic fields seen by the Faraday effect exist on cluster scales in the ICM, or in a mixing layer around the radio plasma which emits the polarised emission (Bicknell et al. 1990; Rudnick \& Blundell 2003). However, there is no valid indication of a source local Faraday effect in the discussed cases (Enßlin et al. 2003), and the Faraday rotation signal excess of radio sources behind clusters compared to a field control sample strongly supports the existence of strong magnetic fields in the wider ICM (Clarke et al. 2001; Johnston-Hollitt \& Ekers 2004; Clarke 2004).

The morphological structure of magnetic fields in galaxy clusters is difficult to obtain due to the projection effects in radio observations. However, there are situations in which the magnetic fields are illuminated in only sheet-like sub-volumes of clusters. This happens whenever short-lived ultra-relativistic electrons are injected at a shock wave travelling through the ICM. The electrons usually lose their energy before the shock wave can travel far away. The electrons thereby produce synchrotron radio emission from a nearly sheet-like volume. This emission likely forms the so-called giant radio relics (Enßlin et al. 1998a; Roettiger et al. 1999a). High resolution radio polarisation maps of the relic in Abell 2256, which is one of the largest radio relics known, reveal that the magnetic fields are organised in filaments or sheets with an aspect ratio of at least 5 (Clarke \& Enßlin 2006). Furthermore, the Faraday rotation map of 3C 465 reveals stripy patterns (Eilek \& Owen 2002), also suggesting the existence of intermittent fields in galaxy clusters. The presence of thermally isolated elongated cool $\mathrm{H}-\alpha$ filaments in the core of the Centaurus cluster is also best understood by the existence of filamentary magnetic fields in that environment (Crawford et al. 2005). 


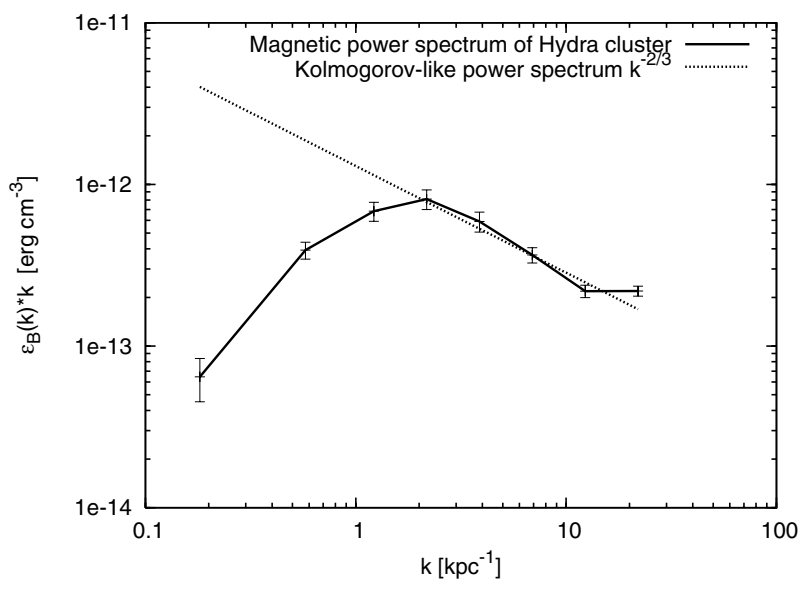

Fig. 1. Magnetic turbulence in the centre of the cool core cluster Hydra A as derived from the Faraday rotation map of the northern radio lobe of Hydra A by Vogt \& Enßlin (2005). An angle of $45^{\circ}$ was assumed in this figure between the line of sight and the approaching north-lobe. Variation of this angle changes the overall normalisation of the spectrum, but not its shape. The right-most data point is likely to be contaminated by observational noise in the Faraday map. A central root-mean-square magnetic field strength of $B_{\mathrm{rms}}=7.3 \pm 0.2 \pm 2 \mu \mathrm{G}$ and a magnetic autocorrelation length of $\lambda_{\mathrm{B}}=2.8 \pm 0.2 \pm 0.5 \mathrm{kpc}$ was derived by Vogt $\&$ Enßlin (2005). The first errors are the statistical uncertainties due to the limited statistics, whereas the systematic error reflects the uncertainties in the geometry of the radio source and the Faraday screen.

\subsection{Structure of the paper}

The paper is structured as follows: in Sect. 2 we introduce the turbulent dynamo and summarise its expected and observed signatures in galaxy clusters, mainly based on the abovementioned estimate of the magnetic power-spectrum in the Hydra A cluster core. In Sect. 3 we develop a steady-state analytical description of the expected magnetic turbulence due to the stirring motion of buoyant radio bubbles from the central galaxy, which is assumed to regulate the energy content of the cool core. This is applied in Sect. 4 to a number of prominent cool core clusters, and compared to existing information on magnetic fields whenever available. Section 5 contains our conclusions.

Throughout the paper, we assume a Hubble constant of $H_{0}=$ $70 \mathrm{~km} \mathrm{~s}^{-1} \mathrm{Mpc}^{-1}$, and translate literature values of luminosities, length scales, electron densities and magnetic field strengths derived from Faraday rotation measurements to this value.

\section{Turbulent magnetic dynamo}

\subsection{Dynamo concepts}

The Kolmogorov-like magnetic power spectrum in the cool core of the Hydra A cluster indicates that the magnetic fields are shaped and probably amplified by hydrodynamical turbulence (e.g. De Young 1992). Therefore, it seems most promising to seek the origin of the observed magnetic power spectrum in the theories of turbulent dynamos. A very similar view and approach are used in the recent work by Subramanian et al. (2006), in which the magnetic fields in non-cooling core clusters were also assumed to be maintained by turbulence. In that environment the turbulence is due to merger events and galaxy motion, whereas here it is due to the inflation and buoyant motion of radio bubbles.

Since cool cores of galaxy clusters are not believed to rotate, the gas flow is probably non-helical and the galactic dynamo theories do not apply here. Instead, the non-helical turbulent dynamo (also called small-scale dynamo) should operate if the gas flow is sufficiently random, as it would be in the case of developed turbulence.

The physical details of the small-scale dynamo are still debated. However, as it turns out, the exact nature of the small scale dynamo is of minor importance for an understanding of cool core magnetic fields. The observed magnetic field is probably determined by the saturated state of such a dynamo, provided the dynamo had sufficient time to amplify seed fields to the dynamically relevant strength. This condition is fulfilled if either the dynamo is very efficient, permitting the very weak primordial fields to be amplified, or if the dynamo can start in an existing, relatively strong magnetisation, which is only a few orders of magnitude below the saturation level. There are arguments in favour of both pre-conditions being fulfilled, which we discuss briefly.

The random gas motion would stretch and fold any initial seed magnetic fields and lead to an exponential growth of the magnetic energy density with time with the characteristic timescale being the eddy-turnover time. This proceeds as long as the dynamical back-reaction of the magnetic field is unimportant. The folding operations of the flow form small-scale magnetic reversals perpendicular to the local field directions (Schekochihin et al. 2002). Magnetic diffusivity limits the scales to be of the order of $\lambda_{\mathrm{B}} \sim \lambda_{\mathrm{T}} R_{\mathrm{m}}^{-1 / 2}$, where $\lambda_{\mathrm{T}}$ is the turbulence injection scale, and $R_{\mathrm{m}}$ is the magnetic Reynolds-number (Ruzmaikin et al. 1989). The typical bending radius of the fields should be of the order of the turbulence length-scale $\lambda_{\mathrm{T}}$.

This picture has been criticised by Goldshmidt \& Rephaeli (1993) as being unable to explain cluster magnetic fields. Their main objection is that the transverse size of the magnetic structures $\lambda_{\mathrm{B}}$ should be extremely small, since the magnetic Reynolds number is typically $R_{\mathrm{m}} \sim 10^{28 \ldots 29}$ in a cluster environment, leading to $\lambda_{\mathrm{B}}$ of the order of a light second. However, magnetic structures with kpc size are required to accommodate the observed Faraday rotation values with physically plausible cluster magnetic fields. This reasoning lead Goldshmidt \& Rephaeli (1993) to argue for a galactic origin of the intra-cluster magnetic fields. Rough estimates of the magnetisation of the intra-cluster medium indicate that galactic outflows in magnetised winds and relativistic plasma jets should lead to a substantial seed magnetisation ${ }^{7}$.

The small-scale dynamo picture can only be reconciled with the observations if the huge magnetic Reynolds number is replaced by a much lower effective value. It was pointed out by Subramanian (1999) that the gas motions induced by the magnetic forces lead to a diminishing of the magnetic field strength, which can be expressed approximately as an effective magnetic diffusivity. This diffusivity increases with growing magnetic field strength, leading to a decreasing effective magnetic Reynolds number permitting the magnetic structures to grow to larger spatial scales. On larger scales more turbulent energy density is available in a Kolmogorov cascade, thus larger field strengths can be accommodated. Larger field strengths imply a further decreased effective magnetic Reynolds number and therefore a further growth of the fields in strength and length scale. This process continues until a saturated state is reached. The magnetic $e$-folding time is of the order of the turbulent eddy time scale and might be sufficient to amplify even

7 Rees (1987), Daly \& Loeb (1990), Chakrabarti et al. (1994), Enßlin et al. (1997, 1998b), Kronberg et al. (1999), Völk \& Atoyan (2000), Kronberg et al. (2001), Bertone et al. (2006). 
primordial magnetic fields in a cluster environment to their observed strength.

It was argued by Schekochihin et al. (2005) that plasma instabilities should lead to an accelerated regime of magnetic amplifications during the pre-saturation phase. Assuming an effective description for the plasma particle pitch angle scattering by the plasma-waves generated in the instabilities, Schekochihin et al. (2005) showed that a nearly explosive production of magnetic fields from very weak primordial seed fields on cosmologically negligible times-scales might be possible.

Thus, although the details of the generation of dynamically relevant magnetic fields in galaxy clusters are still unclear, it seems that there are sufficient sources of magnetisation, and sufficiently efficient dynamo mechanisms present to amplify such fields in cosmologically short times. The question for the interpretation of the observational data is in which state do we expect the fields to be at present. This requires an examination of the saturated dynamo state.

\subsection{Saturated dynamo state}

We start our investigation of the saturated state with the view introduced by Subramanian (1999). As soon as the Lorentz force becomes sufficiently strong, the fields do not follow the flow passively, but try to disentangle themselves. This back-reaction motion leads to an increase in the effective magnetic diffusivity, and therefore to a lower, renormalised magnetic Reynolds number implying that fields become organised on larger scales. The Reynolds number decreases until it reaches a critical value $R_{\text {c }} \sim 10-100$, below which the turbulent dynamo would stop operating. The system reaches a saturated state, in which the magnetic correlation length scale

$\lambda_{\mathrm{B}} \sim \lambda_{\mathrm{T}} R_{\mathrm{c}}^{-1 / 2}$

is solely determined by the turbulence length scale and the critical Reynolds number.

Schekochihin \& Cowley (2006) acknowledge that their explosive dynamo alone fails to explain the observed level of cluster magnetisation, but only by up to one order of magnitude. Therefore, the remaining amplification and the saturated state should result from a conventional non-helical dynamo (e.g. Subramanian 1999). However, Schekochihin \& Cowley (2006) speculate that the physics of the plasma instability driven dynamo still imprints on the saturated state, leading to a characteristic field strength with $\lambda_{\mathrm{B}} \sim \lambda_{\mathrm{T}}\left(v_{\text {th,i }} / v_{\mathrm{T}}\right)^{1 / 2}\left(\rho_{\mathrm{i}} / \lambda_{\mathrm{T}}\right)^{1 / 8}$. Here, $v_{\text {th }}$ and $v_{\mathrm{T}}$ are the ion and the turbulent velocity, respectively. $\rho_{\mathrm{i}}$ is the gyroradius of an ion within a magnetic field that is in equipartition with the turbulent energy density. This picture of the saturated state can be mathematically mapped onto the model of Subramanian (1999) if we define an effective magnetic Reynolds number of

$R_{\mathrm{c}}^{*} \sim \frac{v_{\mathrm{T}}}{v_{\mathrm{th}, \mathrm{i}}}\left(\frac{\lambda_{\mathrm{T}}}{\rho_{\mathrm{i}}}\right)^{\frac{1}{4}}$

which takes values in the range $10^{2 \ldots 3}$ for cluster environments.

In the picture initially developed by Batchelor (1950); Kazantsev (1967); Zeldovich et al. (1990), the magnetic fields are highly intermittent: only a small fraction $f_{\mathrm{B}}$ of the volume is actually strongly magnetised, whereas the remaining volume $1-f_{\mathrm{B}}$ does not carry dynamically important magnetic fields. Zeldovich et al. (1990) and others (Ruzmaikin et al. 1989;
Sokolov et al. 1990; Subramanian 1999) assumed the magnetised regions to be organised as magnetic flux ropes of volume $\lambda_{\mathrm{T}} \lambda_{\mathrm{B}}^{2}$ per eddy volume $\lambda_{\mathrm{T}}^{3}$. The occupied volume fraction would be $f_{\mathrm{B}} \sim \lambda_{\mathrm{B}}^{2} / \lambda_{\mathrm{T}}^{2} \sim R_{\mathrm{c}}^{-1}$. Such highly filamentary structures were not confirmed by numerical simulations. By using a spectral MHD code, Cho \& Vishniac (2000) find for example that the magnetic autocorrelation functions exhibit only a moderate anisotropy on scales where the magnetic energy density peaks. However, numerical simulations in physical space indicate that the magnetic structures are more sheet-like with thickness $\lambda_{\mathrm{B}}$ (Brandenburg \& Subramanian 2004; Schekochihin $\&$ Cowley 2006), leading to a $f_{\mathrm{B}} \sim \lambda_{\mathrm{B}} / \lambda_{\mathrm{T}} \sim R_{\mathrm{c}}^{-1 / 2}$ as assumed in Subramanian et al. (2006). Furthermore, as we explained in Sect. 1.2, radio observations of the cluster radio relic in Abell 2256 also strongly support the assumption of intermittent magnetic fields, probably of sheet-like structure.

In order to allow for any geometry of the magnetic structures in the saturated state, we assume that they are effectively $d$-dimensional, giving

$f_{\mathrm{B}} \sim \frac{\lambda_{\mathrm{T}}^{d} \lambda_{\mathrm{B}}^{3-d}}{\lambda_{\mathrm{T}}^{3}} \sim R_{\mathrm{c}}^{-\frac{3-d}{2}}$, with $1 \leq d \leq 3$.

Under the steady-state conditions of the saturated dynamo, the hydrodynamical dissipation of turbulent energy on scale $\lambda_{\mathrm{T}}$ and the relaxation of magnetic structures bent on the same scales $\lambda_{\mathrm{T}}$ should have the same time scales:

$$
\begin{aligned}
& \tau_{\mathrm{B}} \sim \frac{\lambda_{\mathrm{T}}}{\mathrm{v}_{\mathrm{A}}^{\text {struct. }}}=\lambda_{\mathrm{T}} \sqrt{\frac{\rho}{2 \varepsilon_{B}^{\text {struct. }}}} \\
& \tau_{\mathrm{T}} \sim \frac{\lambda_{\mathrm{T}}}{v_{\mathrm{T}}}=\lambda_{\mathrm{T}} \sqrt{\frac{\rho}{2 \varepsilon_{\mathrm{T}}}},
\end{aligned}
$$

where $\mathrm{v}_{\mathrm{A}}^{\text {struct. }}$ and $\varepsilon_{B}^{\text {struct. }}$ are the Alfvénic velocity and energy density within the magnetic structures, and $v_{\mathrm{T}}$ and $\varepsilon_{\mathrm{T}}$ the turbulent velocity and energy density, respectively. Then $\tau_{\mathrm{B}} \sim \tau_{\mathrm{T}}$ implies that the magnetic fields within the structures are in equipartition with the environmental turbulent energy density, and the volume-averaged magnetic energy density is therefore lower than these by the magnetic volume filling factor:

$\varepsilon_{\mathrm{B}} \sim \varepsilon_{\mathrm{B}}^{\text {struct. }} f_{\mathrm{B}} \sim \varepsilon_{\mathrm{T}} f_{\mathrm{B}} \sim \varepsilon_{\mathrm{T}} R_{\mathrm{c}}^{-\frac{3-d}{2}}$

Thus, with the knowledge or assumption of the critical Reynolds number $R_{\mathrm{c}}$, and the effective dimensionality $d$ of the magnetised regions it is possible to translate properties of the hydrodynamical turbulence to the magnetic turbulence and vice-versa, under the assumption that the system is in the saturated dynamo state.

In the numerical examples, we will investigate three scenarios, which differ in assumed magnetic topology and critical magnetic Reynolds number:

1. Magnetic fields mostly organise in flux tubes $\left(d=1, R_{\mathrm{c}}=\right.$ $\left.20, \Rightarrow f_{\mathrm{B}}=0.05\right)$. This scenario reflects the original assumption on turbulent magnetic structures by Zeldovich et al. (1990) but modified with the concept of the renormalised Reynolds number (Subramanian 1999). Here we consider it to investigate the induced hydrodynamical viscosity on large scales, since there are published predictions for this quantity in the flux rope scenario (Longcope et al. 2003).

2. Magnetic fields mostly organise in sheets and ribbons $(d=$ $\left.2, R_{\mathrm{c}}=35, \Rightarrow f_{\mathrm{B}}=0.17\right)$. This scenario is more in 
agreement with recent numerical simulations, as argued by Subramanian et al. $(2006)^{8}$.

3. The assumed saturated state of the explosive dynamo of Schekochihin \& Cowley (2006), which we characterise by $d=2$ dimensions and by an effective Reynolds number $R_{\mathrm{c}}=R_{\mathrm{c}}^{*}$ provided by Eq. (2).

The scaling of any quantity with the parameters $R_{\mathrm{c}}$ and $d$ will also be provided, so that this theory may be applicable even if the adopted values need readjustment.

\subsection{Magnetic viscosity}

In the saturated dynamo state, the magnetic fields do not passively follow the hydrodynamical flow of the bulk motion, but possess an independent velocity component due to the Lorentz force. This should lead to a slippage of strongly and weakly magnetised regions relative to each other, possibly causing some friction due to induction of small scale eddies in the wakes of the magnetic structures. For the flux rope dynamo scenario, Longcope et al. (2003) give the expected viscosity on large spatial scales in the saturated state to be of the order of $4 \%$ of the turbulent diffusivity $\kappa_{\mathrm{T}} \approx v_{\mathrm{T}} \lambda_{\mathrm{T}} / 3$, thus

$\kappa_{\mathrm{visc}} \approx 0.04 \frac{v_{\mathrm{T}} \lambda_{\mathrm{T}}}{3}$.

Also in the scenario of mainly 2-dimensional magnetic structures, one would expect such viscous effects, but no estimates of the viscosity exist to our knowledge.

\subsection{Signatures}

According to the scenario described above, the magnetic fields in the turbulent cool cores of galaxy clusters should exhibit the following properties:

A. The magnetic power spectrum should either reflect the hydrodynamical power spectrum due to their dynamical coupling, just with a lower normalisation and on smaller spatial scales or the small-scale magnetic fluctuations are expected to follow a Goldreich-Sridar law, which is indistinguishable from a Kolmogorov spectrum in the isotropic average (Goldreich \& Sridhar 1997). That means that a Kolmogorovinertial range power law behaviour of hydrodynamical turbulence should also be found in the magnetic spectrum in any scenario.

B. The average magnetic energy density $\varepsilon_{\mathrm{B}}$ is lower than the turbulent kinetic energy density $\varepsilon_{\text {kin }}$ by $\varepsilon_{\mathrm{B}} \approx \varepsilon_{\text {kin }} f_{\mathrm{B}}$, where $f_{\mathrm{B}} \sim R_{\mathrm{c}}^{-(3-d) / 2} \sim 0.05 \ldots 0.2$ depending on the critical magnetic Reynolds number and the dimensionality of the magnetic structures.

\footnotetext{
${ }^{8}$ Haugen et al. (2004) numerically investigate magnetic turbulence in the case of a Prandtl number of order unity, and find a magnetic to turbulent energy ratio of the order of $f_{\mathrm{B}} \approx 0.4$ in this regime. Since they report a $R_{\mathrm{c}} \approx 35$, their case could be described by $d=2.5$ dimensional magnetic intermittency. However, as Dennis \& Chandran (2005) point out, the dissipation rate of turbulent energy seems to be doubled compared to not (or less) magnetised scenarios, which would lead to a reduction in the turbulent and magnetic energy densities by a similar factor. For the observables of the magnetic fields, like field strength, length and Faraday signal, there would be very little numerical difference to the $d=2$ scenario. For this reason, we do not follow this scenario separately, but assume it to be subsumed under the $d=2$ case.
}

C. The magnetic fluctuations are concentrated on a perpendicular scale $\lambda_{\mathrm{B}}$, which is smaller than the hydrodynamical turbulence injection scale $\lambda_{\mathrm{T}}$ by $\lambda_{\mathrm{B}} \approx \lambda_{\mathrm{T}} R_{\mathrm{c}}^{-1 / 2}$.

D. Magnetic correlations exist up to a scale $\lambda_{\mathrm{T}}$, turn there into an anti-correlation as a consequence of $\boldsymbol{\nabla} \cdot \boldsymbol{B}=0$, and quickly decay on larger scales.

E. The fields may be spatially intermittent. This may be understood by Zeldovich's flux rope model, in which magnetic ropes with diameter $\lambda_{\mathrm{B}}$ are bent on scales of the order $\lambda_{\mathrm{T}}$. Alternatively, as also supported by recent numerical simulations, it can be understood in terms of magnetic sheets of thickness $\lambda_{\mathrm{B}}$ and size $\lambda_{\mathrm{T}}$.

F. In the case of intermittent fields, the field strength within the magnetic structures should be in energy equipartition with the average turbulent kinetic energy density of their environment.

G. The magnetic drag of such intermittent structures produces a hydrodynamical viscosity on large scales. In case of the Zeldovich-flux rope scenario, an estimate of a viscosity of $4 \%$ of the turbulent diffusivity $\kappa_{\mathrm{T}} \approx v_{\mathrm{T}} \lambda_{\mathrm{T}} / 3$ was made by Longcope et al. (2003).

\subsection{Observations}

We investigate briefly if the above predictions of the non-helical dynamo theory are in agreement with observations.

A. A Kolmogorov-like magnetic power spectrum in a cluster cool core is revealed by the Faraday rotation map of Hydra A (Vogt \& Enßlin 2005).

B. Translating the Faraday rotation based estimate of the magnetic energy density to the expected turbulent energy density $\varepsilon_{\mathrm{T}} \sim \varepsilon_{\mathrm{B}} / f_{\mathrm{B}}$ in the Hydra A cluster yields $0.3 \ldots 1.0 \times$ $10^{-10} \mathrm{erg} \mathrm{cm}^{-3}$, which corresponds to turbulent velocities of $v_{\text {turb }} \approx 250 \ldots 430 \mathrm{~km} \mathrm{~s}^{-1}$. This is comparable to velocities of buoyant radio plasma bubbles (Enßlin \& Heinz 2002), which are expected to stir up turbulence (e.g. Churazov et al. 2001).

C. The expected turbulence injection scale in the Hydra A cluster core is of the order of $\lambda_{\mathrm{T}} \sim \lambda_{\mathrm{B}} R_{\mathrm{c}}^{1 / 2} \sim 10 \ldots 20 \mathrm{kpc}$, again consistent with the radio plasma of Hydra A being the source of turbulence, since the turbulence injection scale and the radio lobes of Hydra A have comparable dimensions. The dynamical connection of the radio source length scale and the magnetic turbulence scale would explain why the Faraday map of Hydra A is conveniently sized to show us the peak of the magnetic power spectrum (see Fig. 1).

D. The expectation of weak magnetic fluctuations on scales larger than the turbulence injection scale is hard to test with the available data due to the limited size of the $R M$ map used. However, the downturn of the magnetic power spectrum at small $k$-values visible in Fig. 1 is in good agreement with the requirement of magnetic anti-correlations on large scales.

E. Magnetic structures in the form of flux ropes or ribbons might have been detected as striped patterns in the $R M$ map of 3C 465 (Eilek \& Owen 2002), and as polarised synchrotron filaments in the cluster radio relic in Abell 2256 (Clarke \& Enßlin 2006), in support of the assumed intermittence of the small-scale dynamo.

F. The fraction of the strongly magnetised volume may be as small as $f_{\mathrm{B}}=R_{\mathrm{c}}^{-1} \approx 0.05 \ldots 0.2$. Strongly intermittent fields in galaxy clusters may help to reconcile the discrepancy between Faraday and inverse Compton based magnetic field estimates (Enßlin et al. 1999). The latter estimate could easily be biased to low field regions due to the faster removal 
of ultra-relativistic electrons in strong field regions by synchrotron emission.

G. The expected hydrodynamical viscosity on large scales in the Hydra cluster is of the order of $10^{28} \mathrm{~cm}^{2} / \mathrm{s}$ (if the estimate in Eq. (7) based on the flux rope picture is applicable). Fabian et al. (2003b) argue, for the comparable Perseus cluster cool core, for a lower limit on the large-scale viscosity of $4 \times 10^{27} \mathrm{~cm}^{2} / \mathrm{s}$. They base their arguments on the observation of very elongated $\mathrm{H}-\alpha$ filaments which suggest a laminar flow pattern behind buoyantly rising radio bubbles. An upper limit on the viscosity in the (non-cooling core) Coma cluster of $\sim 3 \times 10^{29} \mathrm{~cm}^{2} / \mathrm{s}$ is reported by Schuecker et al. (2004). Both limits are consistent with our coarse estimate of the large scale viscosity and enclose it. However, the presence of a significant viscosity, as well as the underlying magnetic intermittency, in galaxy clusters should be regarded as being speculative, and not directly confirmed by observations. Nevertheless, there is a growing number of theoretical investigations ${ }^{9}$ on the possibility that viscosity helps to explain properties of the cluster gas.

\section{Magneto-hydrodynamic turbulence in cool cores}

\subsection{Scope of the approach}

A theoretical model of the magneto hydrodynamic turbulence in cool cores of galaxy clusters is developed in the following. The model is intended to capture the most essential features of the physical picture. We do not attempt to make accurate numerical predictions, but hope to get insight into the scaling relations of the different quantities and their order of magnitude values.

Although cool cores exhibit a structured gas distribution, for our order-of-magnitude calculation we describe them as quasihomogeneous spheres. We briefly present a radial extension of our model in Sect. 3.7.

The episodic injection of radio plasma by the central galaxy into cool cores should lead to temporal variations of the state of the core. Nevertheless, we treat the system as being in a steady state. Our estimates provide therefore only approximate temporal mean values. Observations of cool cores will always show snapshots of the cool core evolution. Thus, deviations between our predictions and the observed state of individual cool cores should not be too surprising.

Detailed numerical modelling of the processes would be required to overcome our simplifying assumptions which is well beyond the scope of this initial investigation of the scenario presented.

\subsection{A simplified cool core description}

A cool core is a condensation of cold gas of mass $M_{\mathrm{cc}}$, which dropped out of the hot phase of a galaxy cluster due to the faster radiative cooling of denser gas. The observables of the cool core, which can be used as diagnostics of the physical parameters, are the bolometric X-ray luminosity of the cool core $L_{\mathrm{cc}}$, its temperature $T_{\mathrm{cc}}$, and its radius $r_{\mathrm{cc}}$ from which the central electron density $n_{\mathrm{cc}}$ can be deduced.

9 Pringle (1989); Churazov et al. (2001); Reynolds et al. (2002); Fujita et al. (2004); Ruszkowski et al. (2004); Dennis \& Chandran (2005); Reynolds et al. (2005); Brüggen et al. (2005b); Kaiser et al. (2005).
The energy feedback of the central radio source prevents the cool core gas from falling below a characteristic temperature ${ }^{10}$ $T_{\mathrm{cc}} \sim \mathrm{keV}$, which is given by the requirement that the atomic line emission does not dominate. Otherwise, in the case of a lower temperature, the gas in the cool core would rapidly condense onto the central galaxy due to a cooling catastrophe driven by emission-lines. The pressure in a cool core is

$P_{\mathrm{cc}}=2 n_{\mathrm{cc}} T_{\mathrm{cc}}$,

where we ignore here and in the following the presence of any element heavier than hydrogen in pressure and mass terms for simplicity. The cool core gas mass is therefore

$M_{\mathrm{cc}}=n_{\mathrm{cc}} m_{\mathrm{p}} V_{\mathrm{cc}}$, where $V_{\mathrm{cc}}=\frac{4 \pi}{3} r_{\mathrm{cc}}^{3}$.

The X-ray luminosity of the cool core is given by

$L_{\mathrm{cc}}=\Lambda_{\mathrm{X}}\left(T_{\mathrm{cc}}\right) n_{\mathrm{cc}}^{2} V_{\mathrm{cc}}$

where

$\Lambda_{\mathrm{X}}\left(T_{\mathrm{cc}}\right)=\Lambda_{0} T_{\mathrm{cc}}^{\frac{1}{2}}+\Lambda_{\text {lines }}\left(T_{\mathrm{cc}}\right)$

consists of a Bremsstrahlung- and a line emission term. The line emission term should be at best comparable to the Bremsstrahlung-term, since otherwise the full core would run into a cooling catastrophe. Therefore, we can ignore the lineterm in our rough estimate

$\Lambda_{\mathrm{X}}\left(T_{\mathrm{cc}}\right) \approx \Lambda_{0} T_{\mathrm{cc}}^{\frac{1}{2}}$

with $\Lambda_{0}=5.96 \times 10^{-24} \mathrm{erg} \mathrm{s}^{-1} \mathrm{~cm}^{3} \mathrm{keV}^{-1 / 2}$ (assuming a metalicity of 0.3 solar).

\subsection{Hydrodynamical turbulence injection}

We assume that the injection from radio galaxies is the dominant heating mechanism. Although radio galaxies inject energy, not all of it is in the form of turbulence. During the initial phase of inflation of a radio bubble there are shocks, which can heat the environment (Heinz et al. 1998; Fabian et al. 2003a). Later, a series of sound waves are produced, which might be dissipated through viscosity in the medium ${ }^{11}$. When a radio bubble raises buoyantly in the cool core atmosphere, the environmental gas flows around it, which leads to injection of kinetic energy from the central radio galaxy into the cool core. The time averaged turbulence power of the source is

$L_{\mathrm{T}}=f_{\mathrm{T}} L_{\mathrm{cc}}=\eta_{\mathrm{T}} L_{\mathrm{rg}}$

where $f_{\mathrm{T}}$ is the fraction of cool core heating by turbulence, $\eta_{\mathrm{T}} \leq 1$ is the efficiency of kinetic energy transfer between the injected radio plasma and the cool core gas, and $L_{\mathrm{rg}}$ is the mechanical luminosity of the radio galaxy ( $P \mathrm{~d} V$ work per time). Churazov et al. (2002) argue that the efficiency factor $\eta_{\mathrm{T}}$ should be of the order of one since the radio plasma loses most of its energy by adiabatic expansion during its buoyant rise through one scale-height of the cluster atmosphere. This energy is transfered mechanically to the kinetic energy of the ICM gas and is finally dissipated as heat. It is controversial whether most of this energy

\footnotetext{
${ }^{10}$ We express temperatures in terms of energies by setting the Boltzman constant to unity $\left(k_{\mathrm{B}}=1\right)$.

11 Pringle (1989); Churazov et al. (2001); Reynolds et al. (2002); Fujita et al. (2004); Ruszkowski et al. (2004); Dennis \& Chandran (2005).
} 
is dissipated within the cool core, or within the outer regions of the cluster. Here, we assume the former and set $\eta_{\mathrm{T}}=f_{\mathrm{T}}=0.5$ also allowing non-turbulent heating (shock waves, sound waves, heat transport). However, we also provide the scaling of our results with these parameters.

The injected kinetic energy is dissipated through a Kolmogorov cascade within an eddy turnover-time

$\tau_{\mathrm{T}} \sim \lambda_{\mathrm{T}} / v_{\mathrm{T}}$,

where $\lambda_{\mathrm{T}}$ and $v_{\mathrm{T}}$ are the turbulence injection scale and turbulence root mean square velocity, respectively. The average turbulent energy of the cool core $E_{\mathrm{T}}=M_{\mathrm{cc}} v_{\mathrm{T}}^{2} / 2$ is therefore

$E_{\mathrm{T}}=L_{\mathrm{T}} \tau_{\mathrm{T}} \approx f_{\mathrm{T}} L_{\mathrm{cc}} \lambda_{\mathrm{T}} / v_{\mathrm{T}}$

yielding

$\frac{v_{\mathrm{T}}^{3}}{\lambda_{\mathrm{T}}}=\frac{2 f_{\mathrm{T}} L_{\mathrm{cc}}}{M_{\mathrm{cc}}}=\frac{2 f_{\mathrm{T}} L_{\mathrm{cc}}}{m_{\mathrm{p}} n_{\mathrm{cc}} V_{\mathrm{cc}}}$.

This would allow the determination of the turbulent velocity and energy density if the turbulent injection scale was known. In the following, we attempt a rough estimate of the expected turbulent length scale $\lambda_{\mathrm{T}}$.

\subsection{Hydrodynamical turbulence injection scale}

In the picture adopted for this work, the turbulence is stirred by the movement of buoyant radio plasma bubbles. The turbulence injection scale should therefore be of the order of the radius $r_{\text {bub }}$ of the bubbles, which we approximate to be spheres. Assuming an ultra-relativistic equation of state for the radio plasma, we find the volume of the bubble as

$V_{\text {bub }}=E_{\text {bub }} /(4 P)$,

where

$E_{\text {bub }}=L_{\mathrm{rg}} \tau_{\text {bub }} / 2=f_{\mathrm{T}} L_{\mathrm{cc}} \tau_{\text {bub }} /\left(2 \eta_{\mathrm{T}}\right)$

is the mechanical energy released by the radio galaxy into a bubble during the time $\tau_{\text {bub }}$ the bubble needs to leave the cool core buoyantly. If the turbulence stirring bubbles were filled mostly by thermal gas, the volume of the bubble would be $V_{\text {bub }}=2 E_{\text {bub }} /(5 P)$, which would make some difference to our estimates, but none that change the order of magnitude of our results.

The time available for the jets to inflate the bubble is its rise time through the cool core

$\tau_{\text {bub }} \sim \frac{r_{\mathrm{cc}}}{v_{\text {bub }}} \sim\left(\frac{r_{\mathrm{cc}}}{r_{\text {bub }}}\right)^{\frac{1}{2}} \frac{r_{\mathrm{cc}}}{2 c_{\mathrm{s}}}$,

and can be estimated from the balance of drag and buoyance forces (e.g. Enßlin \& Heinz 2002).

Combining Eqs. (17)-(19) yields the bubble radius

$r_{\text {bub }}=\left(\frac{3 f_{\mathrm{T}} L_{\mathrm{cc}} r_{\mathrm{cc}}^{3 / 2}}{64 \pi \eta_{\mathrm{T}} c_{\mathrm{s}} P_{\mathrm{cc}}}\right)^{\frac{2}{7}}$,

and thereby the required hydrodynamical turbulence scale $\lambda_{\mathrm{T}} \sim$ $r_{\text {bub. Using this and the derived relations above, the turbulent }}$ velocity is

$$
\begin{aligned}
v_{\mathrm{T}} & =\frac{3^{\frac{1}{21}} \eta_{\mathrm{T}}^{\frac{1}{3}} \Lambda_{0}^{\frac{3}{7}} n_{\mathrm{cc}}^{\frac{3}{7}} r_{\mathrm{cc}}^{\frac{3}{7}} T_{\mathrm{cc}}^{\frac{1}{14}}}{2^{\frac{4}{21}} 5^{\frac{1}{21}} f_{\mathrm{T}}^{\frac{1}{3}} m_{\mathrm{p}}^{\frac{2}{7}}} \\
& =173 \mathrm{~km} \mathrm{~s}^{-1}\left(\frac{\eta_{\mathrm{T}}}{f_{\mathrm{T}}}\right)^{\frac{1}{3}}\left(\frac{n_{\mathrm{cc}}}{0.1 \mathrm{~cm}^{-3}}\right)^{\frac{3}{7}}\left(\frac{r_{\mathrm{cc}}}{10 \mathrm{kpc}}\right)^{\frac{3}{7}}\left(\frac{T_{\mathrm{cc}}}{\mathrm{keV}}\right)^{\frac{1}{14}},
\end{aligned}
$$

and thus, the turbulent energy density is given by

$$
\varepsilon_{\mathrm{T}}=\frac{3^{\frac{2}{21}} \eta_{\mathrm{T}}^{\frac{2}{3}} \Lambda_{0}^{\frac{6}{7}} m_{\mathrm{p}}^{\frac{3}{7}} n_{\mathrm{cc}}^{\frac{13}{7}} r_{\mathrm{cc}}^{\frac{6}{7}} T_{\mathrm{cc}}^{\frac{1}{7}}}{2^{\frac{29}{21}} 5^{\frac{2}{21}} f_{\mathrm{T}}^{\frac{2}{3}}}
$$

Table 1 contains the values for $v_{\mathrm{T}}, \lambda_{\mathrm{T}}=r_{\text {bub }}$, and $\varepsilon_{\mathrm{T}}$ for a number of prominent cool core clusters, as expected from our steady-state description of cool core turbulence. Deviations due to episodic evolution of cool core turbulence are possible and expected.

\subsection{Magnetic turbulence}

The energy density of the magnetic turbulence $\varepsilon_{\mathrm{B}}$ is lower by a factor $f_{\mathrm{B}} \sim 0.05 \ldots 0.2$ than the kinematic one $\varepsilon_{\mathrm{T}}$. The lengthscale is also smaller by a factor $R_{\mathrm{c}}^{-1 / 2}$. The root-mean-square magnetic field strength in the cool core is therefore given by

$$
\begin{aligned}
B_{\mathrm{rms}} & =\sqrt{8 \pi \varepsilon_{\mathrm{T}} f_{\mathrm{B}}}=\frac{2^{\frac{17}{21}} 3^{\frac{1}{21}} \pi^{\frac{1}{2}} \eta_{\mathrm{T}}^{\frac{1}{3}} \Lambda_{0}^{\frac{3}{7}} m_{\mathrm{p}}^{\frac{3}{14}} n_{\mathrm{cc}}^{\frac{13}{14}} r_{\mathrm{cc}}^{\frac{3}{7}} T_{\mathrm{cc}}^{\frac{1}{14}}}{5^{\frac{1}{21}} f_{\mathrm{T}}^{\frac{1}{3}} R_{\mathrm{c}}^{\frac{3-d}{4}}} \\
& =\left\{\begin{array}{l}
5.6 \mu \mathrm{G}\left(\frac{\eta_{\mathrm{T}}}{f_{\mathrm{T}}}\right)^{\frac{1}{3}}\left(\frac{n_{\mathrm{cc}}}{0.1 \mathrm{~cm}^{-3}}\right)^{\frac{13}{14}}\left(\frac{r_{\mathrm{cc}}}{10 \mathrm{kpc}}\right)^{\frac{3}{7}}\left(\frac{T_{\mathrm{cc}}}{\mathrm{keV}}\right)^{\frac{1}{14}}\left(\frac{R_{\mathrm{c}}}{20}\right)^{-\frac{1}{2}} \\
10 \mu \mathrm{G}\left(\frac{\eta_{\mathrm{T}}}{f_{\mathrm{T}}}\right)^{\frac{1}{3}}\left(\frac{n_{\mathrm{cc}}}{0.1 \mathrm{~cm}^{-3}}\right)^{\frac{13}{14}}\left(\frac{r_{\mathrm{cc}}}{10 \mathrm{kpc}}\right)^{\frac{3}{7}}\left(\frac{T_{\mathrm{cc}}}{\mathrm{keV}}\right)^{\frac{1}{14}}\left(\frac{R_{\mathrm{c}}}{35}\right)^{-\frac{1}{4}}
\end{array}\right.
\end{aligned}
$$

in our flux rope $(d=1)$ and magnetic sheet $(d=2)$ scenarios, respectively. The magnetic autocorrelation length is

$$
\begin{aligned}
\lambda_{\mathrm{B}} & =\frac{\lambda_{\mathrm{T}}}{R_{\mathrm{c}}^{\frac{1}{2}}}=\frac{3^{\frac{1}{7}} \Lambda_{0}^{\frac{2}{7}} m_{\mathrm{p}}^{\frac{1}{7}} n_{\mathrm{cc}}^{\frac{2}{7}} r_{\mathrm{cc}}^{\frac{9}{7}}}{2^{\frac{11}{7}} 5^{\frac{1}{7}} R_{\mathrm{c}}^{\frac{1}{2}} T_{\mathrm{cc}}^{\frac{2}{7}}} \\
& =0.53 \mathrm{kpc}\left(\frac{n_{\mathrm{cc}}}{0.1 \mathrm{~cm}^{-3}}\right)^{\frac{2}{7}}\left(\frac{r_{\mathrm{cc}}}{10 \mathrm{kpc}}\right)^{\frac{9}{7}}\left(\frac{T_{\mathrm{cc}}}{\mathrm{keV}}\right)^{-\frac{2}{7}}\left(\frac{R_{\mathrm{c}}}{20}\right)^{-\frac{1}{2}}
\end{aligned}
$$

\subsection{Predicting an observable: RM dispersion}

Knowing the depth of the Faraday screen $r_{\mathrm{cc}}$, the magnetic field strength $\left\langle B^{2}\right\rangle$ and the magnetic autocorrelation length $\lambda_{\mathrm{B}}$ allows one to predict the expected dispersion of the Faraday rotation measure from a central radio source ${ }^{12}$, assuming an isotropic distribution of magnetic field strengths with the help of Eq. (40) of Enßlin \& Vogt (2003):

$$
\begin{aligned}
\left\langle R M^{2}\right\rangle & =\frac{1}{2} a_{0}^{2} n_{\mathrm{cc}}^{2} r_{\mathrm{cc}} \lambda_{\mathrm{B}}\left\langle B^{2}\right\rangle \\
& =\frac{3^{\frac{5}{21}} \pi a_{0}^{2} \eta_{\mathrm{T}}^{\frac{2}{3}} \Lambda_{0}^{\frac{8}{7}} m_{\mathrm{p}}^{\frac{4}{7}} n_{\mathrm{cc}}^{\frac{29}{7}} r_{\mathrm{cc}}^{\frac{22}{7}}}{2^{\frac{20}{21}} 5^{\frac{5}{21}} f_{\mathrm{T}}^{\frac{2}{3}} R_{\mathrm{c}}^{2-\frac{d}{2}} T_{\mathrm{cc}}^{\frac{1}{7}}} \\
R M_{\mathrm{rms}}^{\exp } & =\left\{\begin{array}{l}
\frac{739}{\mathrm{~m}^{2}}\left(\frac{\eta_{\mathrm{T}}}{f_{\mathrm{T}}}\right)^{\frac{1}{3}}\left(\frac{n_{\mathrm{cc}}}{0.1 \mathrm{~cm}^{-3}}\right)^{\frac{29}{14}}\left(\frac{r_{\mathrm{cc}}}{10 \mathrm{kpc}}\right)^{\frac{11}{7}}\left(\frac{T_{\mathrm{cc}}}{\mathrm{keV}}\right)^{-\frac{1}{14}}\left(\frac{R_{\mathrm{c}}}{20}\right)^{-\frac{3}{4}} \\
\frac{1180}{\mathrm{~m}^{2}}\left(\frac{\eta_{\mathrm{T}}}{f_{\mathrm{T}}}\right)^{\frac{1}{3}}\left(\frac{n_{\mathrm{cc}}}{0.1 \mathrm{~cm}^{-3}}\right)^{\frac{29}{14}}\left(\frac{r_{\mathrm{cc}}}{10 \mathrm{kpc}}\right)^{\frac{11}{7}}\left(\frac{T_{\mathrm{cc}}}{\mathrm{keV}}\right)^{-\frac{1}{14}}\left(\frac{R_{\mathrm{c}}}{35}\right)^{-\frac{1}{2}}
\end{array}\right.
\end{aligned}
$$

\footnotetext{
${ }^{12}$ Background sources should have twice the variance $\left\langle R M^{2}\right\rangle$ given here due to the doubled screen depth.
} 
Table 1. Application of the model to cool cores of the Hydra A (Hydr.), Centaurus (Cent.), Cygnus A (Cygn.), A1985, A2597, 3C 31, Perseus (Pers.), A85, A2199 and Virgo clusters of galaxies. The first part of the table contains the cluster parameters whose values were taken from the literature. References and comments on possible biases of the data are given in Sect. 4 and in detail in Appendix A. The second part describes the expected hydrodynamical turbulence. The third, fourth, and fifth parts describe the expected magnetic turbulence for the scenarios of magnetic flux ropes $\left(d=1, R_{\mathrm{c}}=20\right)$, flux sheets $\left(d=2 R_{\mathrm{c}}=35\right)$, and flux sheets in the explosive dynamo scenario $\left(d=2 R_{\mathrm{c}}=R_{\mathrm{c}}^{*}\right)$, respectively. Numbers are given here to three digits accuracy, irrespective of the fact that the real uncertainties of most quantities are much larger.

\begin{tabular}{|c|c|c|c|c|c|c|c|c|c|c|}
\hline cluster properties & Hydr. & Cent. & Cygn. & A1958 & A2597 & 3 3C 31 & Pers. & A85 & A2199 & Virgo \\
\hline $\begin{array}{ll}n_{\mathrm{cc}} & {\left[10^{-3} / \mathrm{ccm}\right]}\end{array}$ & 56.1 & 80.6 & 153 & 189 & 73.5 & 180 & 50.8 & 30.8 & 33.7 & 151 \\
\hline$T_{\mathrm{cc}}[\mathrm{keV}]$ & 2.7 & 2.2 & 6.5 & 3.7 & 1.3 & 0.7 & 3 & 5.5 & 1.6 & 1 \\
\hline$r_{\mathrm{cc}}[\mathrm{kpc}]$ & 35.5 & 8.57 & 10.7 & 13.4 & 28 & 1.2 & 57 & 45 & 29 & 1.6 \\
\hline$R M_{\mathrm{rms}}^{\mathrm{obs}}\left[\mathrm{m}^{-2}\right]$ & 1350 & 660 & 1200 & 2900 & 1080 & 25 & - & - & - & - \\
\hline$L_{\text {cluster }} \quad\left[10^{44} \mathrm{erg} / \mathrm{s}\right]$ & 4.61 & 0.32 & 1.79 & 6.22 & 6.89 & 0.077 & 11.8 & 8.16 & 3.28 & 0.14 \\
\hline$L\left(<r_{\text {cooling }}\right) / L_{\text {cluster }}$ & 0.52 & 0.41 & 0.49 & - & 0.61 & - & 0.59 & 0.3 & 0.47 & 0.5 \\
\hline$L_{\mathrm{cc}} / L_{\text {cluster }}$ & 0.37 & 0.14 & 0.3 & 0.2 & 0.14 & 0.0045 & 0.51 & 0.18 & 0.078 & 0.005 \\
\hline \multicolumn{11}{|c|}{ hydrodynamical turbulence } \\
\hline$\lambda_{\mathrm{B}} \sim r_{\text {bub }}[\mathrm{kpc}]$ & 7.68 & 1.45 & 1.7 & 2.84 & 7.52 & 0.2 & 13.3 & 7.15 & 5.94 & 0.25 \\
\hline$\varepsilon_{\mathrm{T}}\left[10^{-10} \mathrm{erg} / \mathrm{ccm}\right]$ & 0.29 & 0.16 & 0.76 & 1.27 & 0.35 & 0.12 & 0.37 & 0.13 & 0.089 & 0.11 \\
\hline$v_{\mathrm{T}}\left[\mathrm{km} \mathrm{s}^{-1}\right]$ & 250 & 156 & 244 & 283 & 240 & 87.5 & 295 & 225 & 177 & 94 \\
\hline magnetic turbulence & \multicolumn{10}{|c|}{$d=1 R_{\mathrm{c}}=20-$ flux rope dynamo } \\
\hline $\begin{array}{ll}\lambda_{\mathrm{B}} & {[\mathrm{kpc}]}\end{array}$ & 1.72 & 0.32 & 0.38 & 0.64 & 1.68 & 0.045 & 2.97 & 1.6 & 1.33 & 0.056 \\
\hline$B_{\mathrm{rms}} \quad[\mu \mathrm{G}]$ & 6.06 & 4.55 & 9.78 & 12.6 & 6.68 & 3.8 & 6.82 & 4.04 & 3.34 & 3.74 \\
\hline$\kappa_{\mathrm{visc}}\left[10^{27} \mathrm{~cm}^{2} / \mathrm{s}\right]$ & 7.89 & 0.93 & 1.71 & 3.31 & 7.43 & 0.073 & 16.1 & 6.61 & 4.33 & 0.097 \\
\hline$R M_{\mathrm{rms}}^{\exp }\left[\mathrm{m}^{-2}\right]$ & 1520 & 351 & 1730 & 4010 & 1930 & 91.5 & 2590 & 607 & 400 & 96.9 \\
\hline$R M_{\mathrm{rms}}^{\mathrm{obs}} / R M_{\mathrm{rms}}^{\mathrm{exp}}$ & 0.89 & 1.88 & 0.69 & 0.72 & 0.56 & 0.27 & - & - & - & - \\
\hline magnetic turbulence & \multicolumn{10}{|c|}{$d=2 R_{\mathrm{c}}=35-$ fluctuation dynamo } \\
\hline $\begin{array}{ll}\lambda_{\mathrm{B}} & {[\mathrm{kpc}]}\end{array}$ & 1.3 & 0.25 & 0.29 & 0.48 & 1.27 & 0.034 & 2.25 & 1.21 & 1 & 0.042 \\
\hline$B_{\mathrm{rms}}[\mu \mathrm{G}]$ & 11.1 & 8.36 & 18 & 23.2 & 12.3 & 6.99 & 12.5 & 7.44 & 6.13 & 6.88 \\
\hline$R M_{\mathrm{rms}}^{\exp }\left[\mathrm{m}^{-2}\right]$ & 2430 & 561 & 2770 & 6420 & 3090 & 146 & 4140 & 970 & 640 & 155 \\
\hline$R M_{\mathrm{rms}}^{\mathrm{obs}} / R M_{\mathrm{rms}}^{\exp }$ & 0.55 & 1.18 & 0.43 & 0.45 & 0.35 & 0.17 & - & - & - & - \\
\hline magnetic turbulence & \multicolumn{10}{|c|}{$d=2 R_{\mathrm{c}}=R_{\mathrm{c}}^{*}-$ explosive dynamo } \\
\hline$\overline{R_{\mathrm{c}}}$ & 1060 & 461 & 461 & 922 & 1630 & 308 & 1380 & 541 & 839 & 279 \\
\hline$\lambda_{\mathrm{B}}[\mathrm{kpc}]$ & 0.24 & 0.068 & 0.079 & 0.094 & 0.19 & 0.012 & 0.36 & 0.31 & 0.2 & 0.015 \\
\hline$B_{\mathrm{rms}}[\mu \mathrm{G}]$ & 4.75 & 4.39 & 9.44 & 10.3 & 4.7 & 4.06 & 5 & 3.75 & 2.77 & 4.09 \\
\hline$R M_{\mathrm{rms}}^{\exp }\left[\mathrm{m}^{-2}\right]$ & 443 & 155 & 762 & 1250 & 452 & 49.3 & 659 & 247 & 131 & 54.9 \\
\hline$R M_{\mathrm{rms}}^{\mathrm{obs}} / R M_{\mathrm{rms}}^{\mathrm{exp}}$ & 3.04 & 4.27 & 1.57 & 2.32 & 2.39 & 0.51 & - & - & - & - \\
\hline
\end{tabular}

The two cases correspond to filamentary and sheet-like magnetic structure scenarios, respectively. Here, $a_{0}=e^{3} /\left(2 \pi m_{\mathrm{e}}^{2} c^{4}\right)$ is the usual Faraday rotation constant ${ }^{13}$.

The scaling of the Faraday dispersion, which can be written approximately as $R M_{\mathrm{rms}}^{\mathrm{exp}} \propto n_{\mathrm{cc}}^{2} r_{\mathrm{cc}}^{3 / 2} T_{\mathrm{cc}}^{0}$, is sufficiently similar to that of the bolometric luminosity of the cool core $L_{\mathrm{cc}} \propto n_{\mathrm{cc}}^{2} r_{\mathrm{cc}}^{3} T_{\mathrm{cc}}^{1 / 2}$ and also to the mass deposition rate $\dot{M} \propto$ $L_{\mathrm{cc}} / T_{\mathrm{cc}} \propto n_{\mathrm{cc}}^{2} r_{\mathrm{cc}}^{3} T_{\mathrm{cc}}^{-1 / 2}$ to expect significant correlations, which are indeed observed (e.g. Taylor et al. 2002).

\footnotetext{
${ }^{13}$ Equation (25) differs from the usually used, but inaccurate $R M$ dispersion formula, which is based on the cell model for the magnetic fields

$\left\langle R M^{2}\right\rangle \approx \frac{1}{3} a_{0}^{2} n_{\mathrm{cc}}^{2} r_{\mathrm{cc}} \lambda_{R M}\left\langle B^{2}\right\rangle$

The proper magnetic autocorrelation length $\lambda_{\mathrm{B}}$ is used in Eq. (25), which differs in a non-trivial way - since this is magnetic powerspectrum dependent - from the $R M$ autocorrelation length $\lambda_{R M}$ derived from $R M$ maps. Usually, $\lambda_{R M}>\lambda_{\mathrm{B}}$. Second, the numerical factor $\frac{1}{2}$ in Eq. (25) properly takes account of the effect of the constraint $\boldsymbol{\nabla} \cdot \boldsymbol{B} \stackrel{2}{=} 0$, whereas for the above equation, uncorrelated patches with internally constant magnetic fields were assumed, which do not have $\boldsymbol{\nabla} \cdot \boldsymbol{B}=0$ at the patch boundaries. Due to the differences, published magnetic field values based on the above equation do not need be accurate, although the two errors partly compensate each other.
}

\subsection{Approximate treatment of the cool core structure}

In the following, we generalise the simplified geometry of a homogeneous cool core to one which has a radial structure. This generalisation allows us to predict the radial dependence of the various quantities and to test if our predictions of the central $R M$ dispersion are expected to change significantly due to the contribution from radii outside the cool core radius.

In order to provide a radius dependent geometry, we make an additional assumption about the radial balance between heating and cooling. We assume that the cool core is settled in an hydrodynamical state, where heating and cooling are balanced locally at all radii below the cooling radius. Note that this does not necessarily need to be true, since in a convecting system the location of heat deposition and radiative cooling can differ, but for the sake of simplicity, we use this approximation.

The cool core is divided into spherical shells of thickness $\mathrm{d} r$, which have a volume $\mathrm{d} V(r)=4 \pi r^{2} \mathrm{~d} r$ and a luminosity $\mathrm{d} L(r)=$ $\Lambda_{\mathrm{X}}\left(T_{\mathrm{cc}}\right) n_{\mathrm{cc}}^{2} \mathrm{~d} V(r)$. Our arguments about the balance of turbulent heating and radiative cooling made in Sect. 3.3 can be identically applied to each of these shells individually, leading to a relation equivalent to Eq. (16):

$\frac{v_{\mathrm{T}}^{3}(r)}{\lambda_{\mathrm{T}}(r)}=\frac{2 f_{\mathrm{T}}}{m_{\mathrm{p}} n_{\mathrm{cc}}} \frac{\mathrm{d} L}{\mathrm{~d} V}(r)$.

To derive the local turbulence injection scale, we use again the local characteristic buoyancy time $\tau_{\text {bub }}(r) \sim r / v_{\text {bub }}(r)$ to predict 




Fig. 2. Radial profile of the expected $R M$ dispersion for a line of sight starting in the midplane of a $\beta$-profile cool core, but displaced from the cluster centre by $x_{\perp}$. The $R M$ dispersion is given in units of our result $R M_{\mathrm{rms}}^{\mathrm{exp}}$ in Eq. (25) for the solid sphere model of the cool core.

the bubble size at any cluster radius $r>r_{\mathrm{cc}}$. For $r \leq r_{\mathrm{cc}}$, we use $r_{\mathrm{cc}}$ to treat the central flat density profile properly. In doing so, we find that the replacement

$r_{\mathrm{cc}} \rightarrow \max \left(r_{\mathrm{cc}}, r\right)$

in any former equation describing local properties as in Eqs. (20)-(25) gives the appropriate function of radius $r$. Of special interest may be the expected radial scaling of the typical magnetic field strength: $B_{\mathrm{rms}} \propto n_{\mathrm{e}}^{13 / 14}(r) r^{3 / 7}$ which is usually a decreasing function of the radius due to the steepness of the electron profiles. For example for $n_{\mathrm{e}} \propto r^{-2}$ (and $T(r) \approx$ const.) we find $B_{\mathrm{rms}} \propto r^{-10 / 7} \propto n_{\mathrm{e}}^{5 / 7}$ which lies well within the range of usually assumed magnetic field scaling with electron density $B_{\mathrm{rms}} \propto n_{\mathrm{e}}^{0.5 \ldots 1}$ (e.g. Dolag et al. 2001).

Global quantities like the energy content, the luminosity of a volume, etc. are calculated by performing the appropriate volume integrals. The increase of the Faraday dispersion along the line of sight should therefore be given by the differential analogy to Eq. (25)

$$
\begin{aligned}
\frac{\mathrm{d}\left\langle R M^{2}\right\rangle}{\mathrm{d} l} & =\frac{1}{2} a_{0}^{2} n_{\mathrm{cc}}^{2} \lambda_{\mathrm{B}}\left\langle B^{2}\right\rangle \\
& =\frac{3 \frac{5}{21} \pi a_{0}^{2} \eta_{\mathrm{T}}^{\frac{2}{3}} \Lambda_{0}^{\frac{8}{7}} m_{\mathrm{p}}^{\frac{4}{7}} n_{\mathrm{e}}^{\frac{29}{7}}(r) \max ^{\frac{15}{7}}\left(r_{\mathrm{cc}}, r\right)}{2^{\frac{20}{21}} 5^{\frac{5}{21}} f_{\mathrm{T}}^{\frac{2}{3}} R_{\mathrm{c}}^{2-\frac{d}{2}} T_{\mathrm{e}}^{\frac{1}{7}}(r)},
\end{aligned}
$$

which reduces to the former result given by Eq. (26) if integrated over a cool core sphere with constant properties within $r_{\mathrm{cc}}$.

Equation (30) allows us to check under which conditions our constant core model gives appropriate results, and under which conditions the $R M$ contributions from larger radii become essential.

For an electron density profile described by the usual $\beta$-model,

$n_{\mathrm{e}}(r)=n_{\mathrm{cc}}\left(1+\frac{r^{2}}{r_{\mathrm{cc}}^{2}}\right)^{-\frac{3}{2} \beta}$,

we find that the contribution to the $R M$ dispersion per logarithmic radius peaks around the core radius $r_{\mathrm{cc}}$ if $\beta>0.25$ (a similar statement can be made for the cool core dominating the X-ray emissivity if $\beta>0.5$ ). Since typical cluster electron density profiles are described by $\beta \geq 0.5$, our ignorance of contributions of outer regions to the $R M$ dispersion only introduces a moderate error of the order of $20-40 \%$ (depending on $\beta$ ) for lines of sight starting in the cluster centre. Off-centre lines of sight, which often occur for extended radio lobes in our sample, will result in significantly lower $R M$ dispersions. This can be seen in Fig. 2, where numerically estimated $R M$-profiles are shown.

Typical pairs of radio lobes are not located in the midplane of the cluster. One lobe has a shorter and one has a longer line-ofsight through the Faraday-active medium. In a spherical geometry of the cluster gas, the additionally accumulated $\left\langle R M^{2}\right\rangle$ of the back lobe, and the missing of the front lobe should compensate roughly in an average over both lobes, provided both lobes are in mirror symmetric positions with respect to the cluster centre. Therefore, in such a case the midplane $R M$ dispersion is a good reference point.

\section{Application to cool cores}

In the following, we apply our model to a number of cool core clusters. The input parameters of our calculations are the central electron density $n_{\mathrm{cc}}$, the central gas temperature $T_{\mathrm{cc}}$, and the core radius $r_{\mathrm{cc}}$ of the cool core. The numbers are taken from the literature and the corresponding references are given in the sections refering to individual clusters in the Appendix A, together with detailed discussions of the individual datasets. The cluster parameters and the derived properties are summarised in Table 1.

As a consistency check for the radii of the cool cores used, we calculate the emissivity $L_{\mathrm{cc}}$ within the cool core according to Eq. (10) and compare this to the reported bolometric $\mathrm{X}$-ray luminosity of the complete cluster $L_{\text {cluster }}$. The fraction of X-ray luminosity due to the cool core is in the range of $L_{\mathrm{cc}} / L_{\text {cluster }}=1 \%-50 \%$. The typical fractional luminosity within the cooling radius, which is defined by the gas having a cooling time less than the Hubble time, was estimated by Peres et al. (1998). Since the cooling radius is larger than the core radius of the cool core, a systematic difference is expected, and indeed found since $L\left(<r_{\text {cooling }}\right) / L_{\text {cluster }}=40 \%-60 \%$ according to Peres et al. (1998). The large differences for some clusters between the ratios are due to shallow electron density profiles with $\beta \approx 0.5$ (see Sect. 3.7).

The other quantities in Table 1 are calculated according to the formulae given in Sect. 3. Note that our expected $R M_{\mathrm{rms}}^{\mathrm{exp}}$ is calculated for a polarised synchrotron source in the middle of the cool core, whereas the real radio emitting volume may be displaced due to an inclination between radio jet and line-ofsight, and/or due to a non-central position. This will cause some deviation of our expectations from the observations and usually biases the observational values to be lower.

For a sample of cool cores with luminosities between $10^{43}-10^{45} \mathrm{erg} / \mathrm{s}$, we predict turbulent velocities in the range $100-300 \mathrm{~km} \mathrm{~s}^{-1}$, magnetic field strengths in the range $3-13 \mu \mathrm{G}$ ( $1-d$ scenario) or $6-23 \mu \mathrm{G}(2-d$ scenario), and Faraday dispersions in the range $100-4000 \mathrm{~m}^{-2}(150-6000$ in the $2-d$ scenario). These values should be compared to existing Faraday rotation measurements, field strength estimates, and future X-ray-spectroscopically determined velocity dispersions, as done in Table 1, and Figs. 3 and 4. We further list our expectations for the large-scale magnetic viscosity, which is speculative, and which were estimated using the calculations of Longcope et al. (2003) for an assumed $1-d$ flux-rope picture of the magnetic field configuration.

To summarise our results, we find that both the one and two dimensional scenarios for the magnetic structures predict rotation measures which are of the same order of magnitude as the 


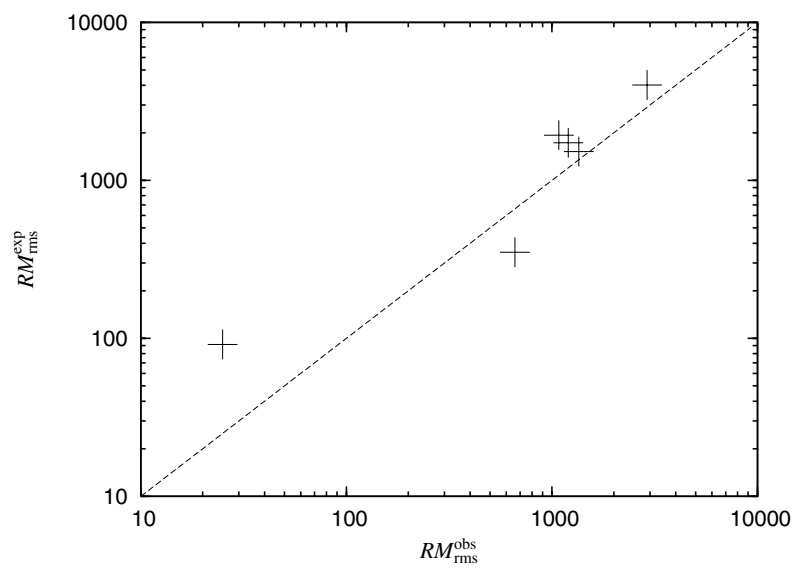

Fig. 3. Comparison of observed (cross) and theoretically expected (case $d=1, R_{\mathrm{c}}=20$ ) (dashed line) root-mean-squared $R M$ signal. The expected $R M$ is calculated for a radio source located at the centre of the cluster, whereas most $R M$ measurements are somewhat off-centre, probably leading to a reduced observed signal.



Fig. 4. Expected (case $d=1, R_{\mathrm{c}}=20$ ) and observed $R M$ dispersion values versus the bolometric cluster luminosity. A linear correlation is also shown to guide the eye.

observed ones. In cases where observational estimates for field strength and length scales existed, there is also a better than order of magnitude agreement. The explosive dynamo seems to underpredict the observed Faraday rotation by a factor of two, due to the shorter magnetic length scale predicted. In general, we would not expect our model to be more accurate than to within a factor of two. This is especially true for our rough parametrisation of the saturation state of the explosive dynamo.

\section{Conclusion}

We showed that many properties of magnetic field measurements in cool core clusters, and especially in the case of Hydra A, which we investigate in detail, seem to support the picture that a saturated small-scale turbulent dynamo is maintaining the magnetic fields. Although there exist dramatic differences between small-scale dynamo scenarios, their saturated state might be quite similar. If it can be assumed to be mostly a balance between the turbulent entangling and Alfvénic straightening of magnetic field lines, the state is characterised by only two numbers: the effective dimensionality of the magnetic structures and the ratio between turbulence injection scale and magnetic correlation length. Casting three representative small-scale dynamo descriptions into this formulation, we find that they are all in rough agreement with the data, which is as much as we can expect given the approximate nature of our treatment. The investigated scenarios are the flux rope dynamo (e.g. Zeldovich et al. 1990; Subramanian 1999, with a renormalised Reynolds number), the fluctuation dynamo as described in Subramanian et al. (2006), and the explosive dynamo introduced by Schekochihin et al. (2005).

The likely energy sources of the expected turbulence are buoyant radio bubbles from the central galaxy, which can inject turbulence with the right amount of power, and also on length scale, that fit the observed magnetic correlation scales well.

Motivated by these indications of the physical processes in cool core clusters, we developed a steady state scenario for the hydrodynamical and magnetic turbulence, assuming that the turbulent feedback from the central radio source compensates for the cool core radiative losses. This scenario predicts turbulent length scales and energy densities that can be compared to observations.

The hydrodynamical turbulence could have been probed with the Astro-E2 satellite mission which unfortunately failed. We hope that successor missions will permit the determination of the gas velocity dispersion (Sunyaev et al. 2003; Inogamov \& Sunyaev 2003; Brüggen et al. 2005a). At present, the magnetic turbulence can be tested with the existing Faraday rotation measurements of extended radio sources in cool core clusters. The Faraday dispersion is a combined measure of the magnetic field strength and correlation length.

For a sample of prominent cool core clusters, we calculate the expected hydro- and magnetic-turbulence, and the predicted Faraday dispersion. In cases of existing Faraday measurements, we find that our estimates reproduce the observed magnitude of the dispersion over roughly two orders of magnitude in $R M$ (or four in $\left\langle R M^{2}\right\rangle$ ). On average, our predictions for the models based on the flux rope and the fluctuating dynamo (Subramanian 1999; Subramanian et al. 2006) are a factor of two higher than the measurements, which is not too surprising, since the actual data sample larger cluster radii, whereas our estimates are aimed at a radio source at the cluster centre. The predictions for the Faraday dispersion in the explosive dynamo scenario are a factor of two below the observation as shown by Schekochihin \& Cowley (2006), which is more severe, since geometrical considerations will only enlarge this discrepancy. However, the description of the saturated state of this dynamos is currently more a guess than a precise estimate. Thus, also this model should be regarded as in agreement with the data.

Very little fine tuning went into our model, but this is likely more a coincidence than a proof of the investigated scenario. There are various places in the calculation where factors of order one could have been introduced. One example is the assumption of the bubble radius being exactly the turbulence injection scale. This assumption will require verification by numerical simulations of the hydrodynamics, which is beyond the scope of this initial investigation. Another uncertain area is the adopted parameters of the dynamo theory, which are also not fully settled in the literature.

Nevertheless, we have shown that the straightforward application of current concepts of turbulent magnetic dynamos in combination with the emerging picture of cool core stabilisation via heat injection due to the dissipation of turbulence seeded by radio galaxy feedback leads to expectations for the Faraday rotation signal that match well with the observations. This is the case for a variety of galaxy clusters spanning two orders of magnitude in their X-ray luminosities. Therefore, our picture of cluster cool 
core heating by radio galaxy feedback in combination with ideas about the properties of the saturated state of non-helical, smallscale magnetic dynamos passed a critical test. Our scenario provides a number of testable predictions, especially about the level of fluid turbulence in individual clusters. We hope that future measurements of the plasma properties in galaxy clusters will even permit discrimination between the different small-scale dynamo models.

Acknowledgements. We acknowledge stimulating discussions with $\mathrm{K}$. Subramanian and A. Schekochihin on the theory of small-scale dynamos. We are thankful to R. Laing for providing us with the results on $3 \mathrm{C} 31$ prior to publication, and to V. Springel and E. Churazov for comments on the manuscript.

\section{References}

Allen, S. W. 1995, MNRAS, 276, 947

Allen, S. W., Schmidt, R. W., Fabian, A. C., \& Ebeling, H. 2003, MNRAS, 342, 287

Allen, S. W., Taylor, G. B., Nulsen, P. E. J., et al. 2001, MNRAS, 324, 842

Böhringer, H., Matsushita, K., Churazov, E., Ikebe, Y., \& Chen, Y. 2002, A\&A, 382,804

Batchelor, G. 1950, Proc. R. Soc. London A, 201, 405

Bertone, S., Vogt, C., \& Enßlin, T. A. 2006 [arXiv: astro-ph/0604462]

Bicknell, G. V., Cameron, R. A., \& Gingold, R. A. 1990, ApJ, 357, 373

Brandenburg, A., \& Subramanian, K. 2004, ArXiv Astrophysics e-prints

Brüggen, M., \& Kaiser, C. R. 2001, MNRAS, 325, 676

Brüggen, M., Kaiser, C. R., Churazov, E., \& Enßlin, T. A. 2002, MNRAS, 331, 545

Brüggen, M., Hoeft, M., \& Ruszkowski, M. 2005a, ArXiv Astrophysics e-prints Brüggen, M., Ruszkowski, M., \& Hallman, E. 2005b, ApJ, 630, 740

Carilli, C. L., \& Taylor, G. B. 2002, ARA\&A, 40, 319

Cen, R. 2005, ApJ, 620, 191

Chakrabarti, S. K., Rosner, R., \& Vainshtein, S. I. 1994, Nature, 368, 434

Chandran, B. D. G. 2004, ApJ, 616, 169

Chandran, B. D. G., \& Maron, J. L. 2004, ApJ, 602, 170

Cho, J., Lazarian, A., Honein, A., et al. 2003, ApJ, 589, L77

Cho, J., \& Vishniac, E. T. 2000, ApJ, 538, 217

Churazov, E., Brüggen, M., Kaiser, C. R., Böhringer, H., \& Forman, W. 2001, ApJ, 554, 261

Churazov, E., Sunyaev, R., Forman, W., \& Böhringer, H. 2002, MNRAS, 332, 729

Churazov, E., Forman, W., Jones, C., \& Böhringer, H. 2003, ApJ, 590, 225

Churazov, E., Forman, W., Jones, C., Sunyaev, R., \& Böhringer, H. 2004, MNRAS, 347, 29

Clarke, T. E. 2004, J. Kor. Astron. Soc., 37, 337

Clarke, T. E., \& Enßlin, T. A. 2006, [arXiv:astro-ph/0603166]

Clarke, T. E., Kronberg, P. P., \& Böhringer, H. 2001, ApJ, 547, L111

Crawford, C. S., Allen, S. W., Ebeling, H., Edge, A. C., \& Fabian, A. C. 1999, MNRAS, 306, 857

Crawford, C. S., Hatch, N. A., Fabian, A. C., \& Sanders, J. S. 2005, MNRAS, 363,216

Daly, R. A., \& Loeb, A. 1990, ApJ, 364, 451

David, L. P., Slyz, A., Jones, C., et al. 1993, ApJ, 412, 479

De Young, D. S. 1992, ApJ, 386, 464

Dennis, T. J., \& Chandran, B. D. G. 2005, ApJ, 622, 205

Dolag, K., Bartelmann, M., \& Lesch, H. 1999, A\&A, 348, 351

Dolag, K., Schindler, S., Govoni, F., \& Feretti, L. 2001, A\&A, 378, 777

Dolag, K., Bartelmann, M., \& Lesch, H. 2002, A\&A, 387, 383

Dolag, K., Jubelgas, M., Springel, V., Borgani, S., \& Rasia, E. 2004, ApJ, 606, L97

Dolag, K., Vogt, C., \& Enßlin, T. A. 2005, MNRAS, 358, 726

Donahue, M., Mack, J., Voit, G. M., et al. 2000, ApJ, 545, 670

Dreher, J. W., Carilli, C. L., \& Perley, R. A. 1987, ApJ, 316, 611

Edge, A. C. 2001, MNRAS, 328, 762

Edge, A. C., \& Frayer, D. T. 2003, ApJ, 594, L13

Eilek, J. A., \& Owen, F. N. 2002, ApJ, 567, 202

Enßlin, T. 2004, J. Kor. Astron. Soc., 37, 439

Enßlin, T. A., \& Heinz, S. 2002, A\&A, 384, L27

Enßlin, T. A., \& Vogt, C. 2003, A\&A, 401, 835

Enßlin, T. A., Biermann, P. L., Kronberg, P. P., \& Wu, X.-P. 1997, ApJ, 477, 560

Enßlin, T. A., Biermann, P. L., Klein, U., \& Kohle, S. 1998a, A\&A, 332, 395

Enßlin, T. A., Wang, Y., Nath, B. B., \& Biermann, P. L. 1998b, A\&A, 333, L47

Enßlin, T. A., Lieu, R., \& Biermann, P. L. 1999, A\&A, 344, 409
Enßlin, T. A., Vogt, C., Clarke, T. E., \& Taylor, G. B. 2003, ApJ, 597, 870 Fabian, A. C., Nulsen, P. E. J., \& Canizares, C. R. 1991, A\&A Rev., 2, 191 Fabian, A. C., Sanders, J. S., Allen, S. W., et al. 2003a, MNRAS, 344, L43 Fabian, A. C., Sanders, J. S., Crawford, C. S., et al. 2003b, MNRAS, 344, L48 Feretti, L., Dallacasa, D., Giovannini, G., \& Tagliani, A. 1995, A\&A, 302, 680 Feretti, L., Dallacasa, D., Govoni, F., et al. 1999, A\&A, 344, 472

Fujita, Y., Suzuki, T. K., \& Wada, K. 2004, ApJ, 600, 650

Garrington, S. T., Leahy, J. P., Conway, R. G., \& Laing, R. A. 1988, Nature, 331, 147

Ge, J. P., \& Owen, F. N. 1993, AJ, 105, 778

Goldman, I., \& Rephaeli, Y. 1991, ApJ, 380, 344

Goldreich, P., \& Sridhar, S. 1997, ApJ, 485, 680

Goldshmidt, O., \& Rephaeli, Y. 1993, ApJ, 411, 518

Govoni, F., Taylor, G. B., Dallacasa, D., Feretti, L., \& Giovannini, G. 2001, A\&A, 379, 807

Govoni, F., \& Feretti, L. 2004, Int. J. Mod. Phys. D, 13, 1549

Hansen, L., Jorgensen, H. E., \& Norgaard-Nielsen, H. U. 1995, A\&A, 297, 13

Hardcastle, M. J., Worrall, D. M., Birkinshaw, M., Laing, R. A., \& Bridle, A. H. 2002, MNRAS, 334, 182

Haugen, N. E., Brandenburg, A., \& Dobler, W. 2004, Phys. Rev. E, 70, 016308

Heckman, T. M., Baum, S. A., van Breugel, W. J. M., \& McCarthy, P. 1989, ApJ, 338,48

Heinz, S., Reynolds, C. S., \& Begelman, M. C. 1998, ApJ, 501, 126

Hoeft, M., \& Brüggen, M. 2004, ApJ, 617, 896

Ikebe, Y., Makishima, K., Ezawa, H., et al. 1997, ApJ, 481, 660

Inogamov, N. A., \& Sunyaev, R. A. 2003, Astron. Lett., 29, 791

Jaffe, W. 1980, ApJ, 241, 925

Jaffe, W., \& Bremer, M. N. 1997, MNRAS, 284, L1

Johnston-Hollitt, M., \& Ekers, R. D. 2004 [arXiv: astro-ph/0411045]

Jubelgas, M., Springel, V., \& Dolag, K. 2004, MNRAS, 351, 423

Kaiser, C. R., Pavlovski, G., Pope, E. C. D., \& Fangohr, H. 2005, MNRAS, 359, 493

Kazantsev, A. 1967, J. Exp. Theor. Phys., 53, 1806

Kim, K.-T., Kronberg, P. P., \& Tribble, P. C. 1991, ApJ, 379, 80

Komossa, S., \& Böhringer, H. 1999, A\&A, 344, 755

Kronberg, P. P., Lesch, H., \& Hopp, U. 1999, ApJ, 511, 56

Kronberg, P. P., Dufton, Q. W., Li, H., \& Colgate, S. A. 2001, ApJ, 560, 178

Laing, R. A. 1988, Nature, 331, 149

Loewenstein, M., \& Fabian, A. C. 1990, MNRAS, 242, 120

Longcope, D. W., McLeish, T. C. B., \& Fisher, G. H. 2003, ApJ, 599, 661

Malyshkin, L. 2001, ApJ, 554, 561

Matsushita, K., Belsole, E., Finoguenov, A., \& Böhringer, H. 2002, A\&A, 386, 77

Mazzotta, P., Brunetti, G., Giacintucci, S., Venturi, T., \& Bardelli, S. 2004, J. Kor. Astron. Soc., 37, 381

McNamara, B. R., Wise, M. W., Nulsen, P. E. J., et al. 2001, ApJ, 562, L149

Mohr, J. J., \& Evrard, A. E. 1997, ApJ, 491, 38

Mohr, J. J., Mathiesen, B., \& Evrard, A. E. 1999, ApJ, 517, 627

Narayan, R., \& Medvedev, M. V. 2001, ApJ, 562, L129

Nipoti, C., \& Binney, J. 2004, MNRAS, 349, 1509

O’Dea, C. P., Payne, H. E., \& Kocevski, D. 1998, AJ, 116, 623

Oegerle, W. R., Cowie, L., Davidsen, A., et al. 2001, ApJ, 560, 187

Peres, C. B., Fabian, A. C., Edge, A. C., et al. 1998, MNRAS, 298, 416

Perley, R. A., \& Taylor, G. B. 1991, AJ, 101, 1623

Pfrommer, C., \& Enßlin, T. A. 2004a, A\&A, 413, 17

Pfrommer, C., \& Enßlin, T. A. 2004b, MNRAS, 352, 76

Pollack, L. K., Taylor, G. B., \& Allen, S. W. 2005, MNRAS, 359, 1229

Pringle, J. E. 1989, MNRAS, 239, 479

Quilis, V., Bower, R. G., \& Balogh, M. L. 2001, MNRAS, 328, 1091

Rees, M. J. 1987, QJRAS, 28, 197

Reynolds, C. S., Heinz, S., \& Begelman, M. C. 2002, MNRAS, 332, 271

Reynolds, C. S., McKernan, B., Fabian, A. C., Stone, J. M., \& Vernaleo, J. C. 2005, MNRAS, 357, 242

Roettiger, K., Burns, J. O., \& Stone, J. M. 1999a, ApJ, 518, 603

Roettiger, K., Stone, J. M., \& Burns, J. O. 1999b, ApJ, 518, 594

Roland, J. 1981, A\&A, 93, 407

Rudnick, L., \& Blundell, K. M. 2003, ApJ, 588, 143

Ruszkowski, M., \& Begelman, M. C. 2002, ApJ, 581, 223

Ruszkowski, M., Brüggen, M., \& Begelman, M. C. 2004, ApJ, 615, 675

Ruzmaikin, A., Sokolov, D., \& Shukurov, A. 1989, MNRAS, 241, 1

Salomé, P., \& Combes, F. 2003, A\&A, 412, 657

Sanders, J. S., \& Fabian, A. C. 2002, MNRAS, 331, 273

Sanders, J. S., Fabian, A. C., \& Dunn, R. J. H. 2005, MNRAS, 360, 133

Schekochihin, A., Cowley, S., Maron, J., \& Malyshkin, L. 2002, Phys. Rev. E, 65, 016305

Schekochihin, A. A., \& Cowley, S. C. 2006, ArXiv Astrophysics e-prints Schekochihin, A. A., Cowley, S. C., Kulsrud, R. M., Hammett, G. W., \& Sharma, P. 2005, ApJ, 629, 139 
Schuecker, P., Finoguenov, A., Miniati, F., Böhringer, H., \& Briel, U. G. 2004, A\&A, 426, 387

Smith, E. P., Bohlin, R. C., Bothun, G. D., et al. 1997, ApJ, 478, 516

Soker, N. 2003, MNRAS, 342, 463

Soker, N. 2004, MNRAS, 350, 1015

Soker, N., \& Sarazin, C. L. 1990, ApJ, 348, 73

Soker, N., \& Pizzolato, F. 2005, ApJ, 622, 847

Soker, N., Blanton, E. L., \& Sarazin, C. L. 2004, A\&A, 422, 445

Sokolov, D. D., Ruzmaikin, A. A., \& Shukurov, A. 1990, in Galactic and Intergalactic Magnetic Fields, IAU Symp., 140, 499

Subramanian, K. 1999, Phys. Rev. Lett., 83, 2957

Subramanian, K., Shukurov, A., \& Haugen, N. E. L. 2006, MNRAS, 366, 1437

Sunyaev, R. A., Norman, M. L., \& Bryan, G. L. 2003, Astron. Lett., 29, 783

Taylor, G. B., \& Perley, R. A. 1993, ApJ, 416, 554

Taylor, G. B., Govoni, F., Allen, S. W., \& Fabian, A. C. 2001, MNRAS, 326, 2
Taylor, G. B., Fabian, A. C., \& Allen, S. W. 2002, MNRAS, 334, 769

Tribble, P. C. 1993, MNRAS, 263, 31

Vogt, C., \& Enßlin, T. A. 2003, A\&A, 412, 373

Vogt, C., \& Enßlin, T. A. 2005, A\&A, 434, 67

Vogt, C., Dolag, K., \& Enßlin, T. A. 2005, MNRAS, 358, 732

Voigt, L. M., \& Fabian, A. C. 2004, MNRAS, 347, 1130

Voigt, L. M., Schmidt, R. W., Fabian, A. C., Allen, S. W., \& Johnstone, R. M. 2002, MNRAS, 335, L7

Völk, H. J., \& Atoyan, A. M. 2000, ApJ, 541, 88

White, D. A. 2000, MNRAS, 312, 663

Widrow, L. M. 2002, Rev. Mod. Phys., 74, 775

Zeldovich, Y. B., Ruzmaikin, A. A., \& Sokoloff, D. D. 1990, The almighty chance (World Scientific Lecture Notes in Physics, Singapore: World Scientific Publication) 
T. A. Enßlin and C. Vogt: Magnetic turbulence in cool cores, Online Material p 1

\section{Online Material}




\section{Appendix A: Individual clusters}

In this Appendix the observational data is discussed. This collection of literature values for cool core cluster parameters made use of the work done by Pfrommer \& Enßlin (2004a).

\section{A.1. Hydra A cluster}

The gas parameters for the Hydra A cluster are taken from Mohr \& Evrard (1997), and the X-ray luminosity is provided by Ikebe et al. (1997). The first detailed $R M$ map of Hydra A was published by Taylor \& Perley (1993), which exhibited very large $R M$ values, especially for the south lobe of Hydra A. The data was reanalysed using the PACERMAN algorithm (Dolag et al. 2005; Vogt et al. 2005), which removed all areas in the northern lobe, and many of the southern lobe with extremely high $R M$ values, indicating that those were observational artefacts. Since the southern lobe $R M$ data seem to be still contaminated by observational artefacts, as statistical tests for anti-correlations of gradients of polarisation angles and $R M$ values reveal (Enßlin et al. 2003), we use only the dispersion of the northern lobe, as reported by Vogt et al. (2005). The same $R M$ map was analysed by Vogt \& Enßlin (2005) using a maximum-likelihood power spectra estimator (see Fig. 1), revealing central magnetic field strength of $B_{\mathrm{rms}} \approx 7 \mu \mathrm{G}$ with an autocorrelation length of $\lambda_{\mathrm{B}} \approx 3 \mathrm{kpc}$.

We note that model expectations and observational values of Faraday dispersion, magnetic field strength and autocorrelation length agree well. Since the Hydra A north lobe is known to approach the observer (and the radio emission extend to larger radii), the observational $R M$ dispersion should be slightly biased to lower values.

\section{A.2. Centaurus cluster}

The central electron density and the cool core radius are taken from Mohr et al. (1999) ${ }^{14}$, and the central temperature from White $(2000)^{15}$. The observed Faraday dispersion for the Centaurus cluster is derived from the embedded radio source PKS $1246-410$ by Taylor et al. (2002). The published Faraday rotation map reveals several isolated patches of extreme $R M$ values, and the histogram of $R M$ values exhibits multiple peaks. A statistical analysis of the alignment of $R M$ gradients and polarisation angle gradients reveals a significant anti-correlation between the two (Enßlin et al. 2003), which is also an indication of observational artefacts in this map. Thus, the reported $R M$ dispersion may be too large. However, an order of magnitude agreement between observed and expected $R M$ dispersion is found.

Taylor et al. (2002) report a $R M$-correlation length of $\lambda_{R M} \sim$ $0.7 \mathrm{kpc}$ and a field strength of $9 \mu \mathrm{G}$, which is significantly different to our expectations. The different length scale is not surprising, since usually $\lambda_{R M}>\lambda_{\mathrm{B}}$ (Enßlin \& Vogt 2003). The larger observationally derived magnetic field strength may be a result of the very small core radius and/or the possible overestimate of the $R M$ dispersion reported in Taylor et al. (2002).

Thus, the X-ray and radio observational data of the cool core of the Centaurus cluster would need further improvements be-

\footnotetext{
14 Using the extremely small core radius given in Taylor et al. (2002) leads to a cool core fractional luminosity of only $3 \%$, which is obviously much smaller than the $40 \%$ observed (Peres et al. 1998).

15 Lower central temperatures have been reported by Sanders \& Fabian (2002) and Taylor et al. (2002), but since they were not used for the electron density estimates, and since our magnetic field and $R M$ predictions are quite insensitive to the temperature, we do not use them.
}

fore conclusive statements about an agreement or disagreement of our model and observations can be made in this case. Both the observed and the expected $R M$ dispersion could be subject to re-adjustments. The complex morphology of this cool core probably causes the difficulties singling out a "real" core radius and central electron density.

\section{A.3. Abell 1958 cluster (3C 295)}

The electron density, central temperature, cool core radius, and dispersion measure are taken from Allen et al. (2001) and the cluster luminosity from Allen et al. (2003).

The agreement of predicted and observed Faraday dispersion is very good. Furthermore, the rather high predicted magnetic field strength of $10-20 \mu \mathrm{G}$ is also found observationally, since Allen et al. (2001) report $14 \mu \mathrm{G}$.

\section{A.4. Cygnus A cluster}

We use the electron density, temperature and cool core radius reported in Mohr et al. (1999). We measure the $R M$ dispersion to be $1200 \mathrm{~m}^{-2}$ from the map published by Dreher et al. (1987) after removing pixels from the map with extraordinary large $R M$ jumps, which we associate with observational artefacts ( $n \pi$-ambiguities). Similar to the case of Hydra A, we see an asymmetry in the $R M$ dispersion between the two lobes, which is a consequence of the different depth of the lobes (the Laing-Garrington effect: Laing 1988; Garrington et al. 1988). Unfortunately, the two radio lobes are located well beyond the cool core radius, which is even more true for their polarised regions used for $R M$ measurements. Since we expect a declining magnetic field strength as a function of radius, the reported $R M$ dispersion is likely smaller than the one that would be measured from a radio source located in the centre of the cool core, as discussed in Sect. 3.7 and illustrated in Fig. 2. The predicted central $R M$ dispersion is a factor of two smaller than the peripherally measured one. With our current understanding of the geometry, there is no apparent conflict between these numbers.

\section{A.5. Perseus cluster}

We take the central electron density, temperature and the cool core radius from Churazov et al. (2003) and the cluster X-ray luminosity from David et al. (1993).

The Perseus cluster cool core exhibits diffuse radio emission, a radio mini-halo. One can apply the classical minimum-energy arguments to the radio halo data to search for the minimum of the sum of the magnetic and relativistic particle energy densities necessary to reproduce the radio synchrotron emission, and assume a constant proton to electron ratio $k_{\mathrm{p}}$. Pfrommer $\&$ Enßlin (2004b) report a classical minimum field strength of $7.2_{-0.4}^{+4.5} \mu \mathrm{G}$ (assuming $k_{\mathrm{p}}=1$ ), where the confidence interval is given by the requirement that the energy density should be within one $e$ fold from the minimum. Pfrommer \& Enßlin (2004b) also develop and apply the hadronic minimum-energy criteria to the Perseus mini-halo. This criterion assumes that the relativistic electrons are injected by hadronic interactions of a relativistic proton population, which usually dominates the relativistic energy budget. No proton-to-electron factor $k_{\mathrm{p}}$ has to be assumed in this case, since the physics of the hadronic interaction determines this ratio. Applied to the Perseus radio halo, a very similar central field strength of $8.8_{-5.4}^{+13.8} \mu \mathrm{G}$ was found. Our cool core model predicts a field strength of $7-13 \mu \mathrm{G}$, which is in 
agreement with these findings. A significant lower central field value of about $1-3 \mu \mathrm{G}$ was reported by Sanders et al. (2005), based on an Inverse Compton interpretation of a hard photon component in the X-ray spectra of the cool core region. If the hard photons flux is due to another physical mechanism, the derived value will become a lower limit. If the Inverse Compton nature of the flux could be verified, this will be a very good field estimate in the case of a non-intermittent field distribution. If there is significant magnetic intermittency, spatially inhomogeneous electron cooling can produce an anti-correlation between fields and relativistic electrons, which can easily lead to Inverse Compton based magnetic field estimates lower by a factor of two (Enßlin et al. 1999; Enßlin 2004).

As stated before, Fabian et al. (2003b) argued for a largescale viscosity in the Perseus cluster cool core of at least $4 \times$ $10^{27} \mathrm{~cm}^{2} / \mathrm{s}$, which is in good agreement with the $1.6 \times 10^{28} \mathrm{~cm}^{2} / \mathrm{s}$ predicted in our $1-d$ scenario.

\section{A.6. Abell 2597}

This cool core galaxy cluster has been observed with Chandra by McNamara et al. (2001). The observation has been reanalysed recently by Pollack et al. (2005). The gas parameters are taken from this reanalysis. The total luminosity used is taken from David et al. (1993).

Pollack et al. (2005) study also the polarisation properties of the central radio source in this cluster. They determine a $R M$ dispersion of $1080 \mathrm{rad} \mathrm{m}^{-2}$, comparable to our expectations. They conclude that the cluster magnetic field has a minimum magnetic field strength of $2.1 \mu \mathrm{G}$. Our model predicts a higher magnetic field strength of $7-12 \mu \mathrm{G}$ which is in agreement with the lower limit of Pollack et al. (2005).

\section{A.7. $3 C 31$}

The cool X-ray environment of the extended radio source 3C 31 is associated with a group of galaxies. X-ray measurements suggest that this group shows properties similar to a cool core of a galaxy cluster. Therefore, it seems to be a good case to test our model in a different situation. The parameters for the gas were taken from Hardcastle et al. (2002). However we use the total luminosity from Komossa \& Böhringer (1999).
Detailed $R M$ measurements have been carried out by Laing et al. (in prep.). They determine the $R M$ dispersion to be $25 \mathrm{rad} \mathrm{m}^{-2}$ (Laing, private communication).

\section{A.8. Abell 85}

We use the electron density, temperature, cool core radius, and luminosity reported in Mohr et al. (1999), and David et al. (1993). To our knowledge, no $R M$ measurements or magnetic field estimates are available for the centre of this and all following clusters. Thus, our $R M$ results are predictions.

\section{A.9. Abell 2199}

The parameters of the electron density profile as reported in Mohr et al. (1999) were used. The temperature of the cool core are taken from Voigt et al. (2002) and the total luminosity from David et al. (1993).

\section{A.10. Virgo cluster}

The Virgo cluster was observed with XMM-Newton by Matsushita et al. (2002). The gas parameters are taken from their analysis. However the total luminosity of David et al. (1993) is used for our calculations. The large difference between the cool core luminosity and the luminosity within the (larger) cooling radius is due to the extremely shallow density profile $(\beta \approx 0.5)$ of Virgo. However, this should not affect our RM estimates too much, since only a $\beta \approx 0.25$ should lead to problems, as was argued in Sect. 3.7. 